ESAIM: M2AN 55 (2021) S909-S939

https://doi.org/10.1051/m2an/2020064
ESAIM: Mathematical Modelling and Numerical Analysis

www.esaim-m2an.org

\title{
ON THE LOCKING-FREE THREE-FIELD VIRTUAL ELEMENT METHODS FOR BIOT'S CONSOLIDATION MODEL IN POROELASTICITY
}

\author{
Xialan Tang ${ }^{1,2}$, Zhibin Liu ${ }^{1}$, Baiju Zhang ${ }^{3, *}$ and Minfu Feng ${ }^{3}$
}

\begin{abstract}
We propose and analyze two locking-free three-field virtual element methods for Biot's consolidation model in poroelasticity. One is a high-order scheme, and the other is a low-order scheme. For time discretization, we use the backward Euler scheme. The proposed methods are well-posed, and optimal error estimates of all the unknowns are obtained for fully discrete solutions. The generic constants in the estimates are uniformly bounded as the Lamé coefficient $\lambda$ tends to infinity, and as the constrained specific storage coefficient is arbitrarily small. Therefore the methods are free of both Poisson locking and pressure oscillations. Numerical results illustrate the good performance of the methods and confirm our theoretical predictions.
\end{abstract}

Mathematics Subject Classification. 65M12, 65M60.

Received February 23, 2020. Accepted August 28, 2020.

\section{INTRODUCTION}

Biot's consolidation model describes the interactions between mechanical deformations and fluid flow in a porous media. It's theoretical basis goes back to the work of Terzaghi [57], in which the author analyzed the one-dimensional (1D) consolidation of a soil column under a constant load. Later, this theory was generalized by Biot to the 3D transient consolidation [21,22]. This model has been widely used in various engineering fields including geomechanics, petroleum engineering, and biomedical engineering.

Since analytical solutions of this model are rarely available due to the complex coupled nature of the equations, the study of its numerical solutions has been of great interest. There is extensive literature on numerical methods for Biot's consolidation model. The most commonly used numerical methods are finite element methods based on two-field formulation. A natural choice in this formulation is to make use of a continuous Galerkin method for both the displacement and pressure. However, some studies have shown that such an approach may lead to nonphysical pressure oscillations of numerical solutions, called poroelasticity locking, for certain ranges of material parameters and small time step sizes [53,60,65].

To avoid the poroelasticity locking, various numerical methods for the problem with different formulation were investigated. Murad, Thomée and Loula [45-47] studied the classical two-field formulation of Biot's model

Keywords and phrases. Virtual elements, poroelasticity, Biot's consolidation model, locking-free, pressure oscillations.

1 Southwest Petroleum University State Key Laboratory of Oil and Gas Reservoir Geology and Exploitation, Chengdu 610500, P.R. China.

2 Chengdu North Petroleum Exploration and Development Technology Company Limited, Chengdu 610051, P.R. China.

3 College of Mathematics, Sichuan University, Chengdu 610065, P.R. China.

*Corresponding author: zhangbaiju1990@163.com 
in incompressible porous media using inf-sup stable finite elements for Stokes equations. However, as shown in [54], an inf-sup stable pair of spaces does not necessarily provide oscillation-free solutions. They add a timedependent stabilization term to overcome pressure oscillation. We also refer the reader to other two-field based numerical methods, such as discontinuous Galerkin methods (DG) [32], stabilized finite element methods [30], Hybrid High-Order methods (HHO) [23], weak Galerkin methods (WG) [33,41] and Hybridizable discontinuous Galerkin methods (HDG) [36].

Another commonly used formulation for Biot's model is three-field formulation, in which displacement, fluid flux, and pressure are main unknowns. Phillips and Wheeler [49-51] proposed and analyzed several methods that couple continuous and discontinuous Galerkin (DG) methods for the displacement with a mixed finite element method for the fluid flux and pressure. Later they heuristically analyzed the case of locking in [52] and concluded that the poroelastic locking typically occurs due to the fact that at an early time the porous medium behaves as an incompressible material, when the constrained specific storage coefficient is very close to 0 , the permeability of the porous medium is very low, and a small time step is used. Therefore they suggested using a discrete subspace that contains nonconstant divergence-free vectors for displacement. This theory motivates some nonconforming methods $[42,61]$ for the displacement coupling with mixed finite element methods for other unknowns. Recently, the author in [62] reexamined the cause of pressure oscillations in the three-field mixed finite element method from an algebraic point of view. The author concluded that pressure oscillations occur due to the incompatibility of the spaces for the displacement and pore pressure assuming the flux and pressure spaces satisfy the inf-sup condition. This idea inspired us to apply inf-sup stable virtual element methods to Biot's Model to overcome Poisson locking and pressure oscillations. In addition to the above methods, there are other three-field based numerical methods that can overcome pressure oscillations $[19,40]$.

The virtual element method (VEM), firstly introduced in [9], is one of the high-order discretization schemes which can be seen as an evolution of the Mimetic Finite Difference method (MFD) [11]. This method has attracted considerable attention in the engineering and numerical mathematics community due to its several appealing features. For example, the shape functions of VEM can no longer be polynomial functions so that it is possible to define a family of conforming elements like $H^{\alpha}$-conforming ( $\alpha$ is a positive integer), $H(\operatorname{div})$ conforming and $H$ (curl)-conforming elements on polygonal and polyhedral meshes. Generally, these types of shape functions cannot be written explicitly (that is why it is called "virtual element"), so the related bilinear forms are usually uncomputable. To overcome this issue, VEM use approximated discrete bilinear forms. Such bilinear forms can be computed exactly by the degrees of freedom related to virtual element subspaces and, at the same time, can preserve the polynomial accuracy that one has on simplexes while working on polyhedra. The VEM has been developed successfully in a wide range of problems such as the Poisson's equation [9], the Darcy problem in mixed form [26], the general second order elliptic problems in primal [13] and mixed form [14], the advection-diffusion problems [18], the Stokes and Navier-Stokes problems [3,16,17,43], eigenvalue problems $[29,38,39,44]$. For elasticity problems, we refer to $[5,8,37,63,64]$. For time-dependent problems in VEM context, we refer to $[4,58,59]$. Recently, the authors in [34] designed and studied fully coupled numerical schemes using virtual element method and finite volume method for Biot Equations Modelling, which is based on two-field formulation. Another paper about VEM for Biot equations is [27]. The authors constructed and analysed a new VEM for the Biot equations in three-field formulation which is based on displacements, pore pressure and total pressure.

In the present contribution, we propose and analyze locking-free virtual element methods for Biot's consolidation model in poroelasticity. Differently from [27,34], our methods couple $H^{1}$-conforming VEM introduced in [2] for the displacement with a mixed VEM introduced in [14] for the fluid flux and pressure. For time discretization, we apply the backward Euler scheme. For virtual element method of order $k \geq 2$, we proved that the generic constants in fully discrete error estimates are uniform with respect to Lamé constant $\lambda$ and constrained specific storage coefficient $c_{0}$. Therefore our method not only can overcome Poisson locking, but also avoid the poroelasticity locking. For $k=1$, we observed in numerical experiments that proposed method suffers Poisson locking or pressure oscillation in triangular meshes while it seems stable in other meshes. That is to say, when $k=1$, the stability of the method depends on mesh. In this paper, we are more interested in the method that is 
stable not only in triangular meshes but also in other meshes. Therefore, we develop another low-order virtual element method for the Biot's consolidation model. Such an element is inspired by the works of Fortin [35], and Bernardi and Raugel [20], and has been analyzed in our previous paper [56] for linear elasticity problem. The main idea is to introduce extra degrees of freedom related to the normal component of $\mathbf{v}$ on each edge, so that the related Fortin operator can be easily constructed, which is an important operator in deriving robust error estimates. We also prove that the low order method is free of both Poisson locking and pressure oscillations.

The rest of paper is arranged as follows. In Section 2, we recall the Biot's consolidation model and its variational formulation. In Section 3, we elaborate the proposed virtual element method including the construction of approximation spaces and computable bilinear forms. In Section 4, we introduce the fully discrete scheme and derive error analysis. Finally, Section 5 presents several numerical tests to confirm our theoretical analysis.

We end this section with recalling some useful notations to be used below. We will use standard notation for Sobolev spaces and norms (see [1] for more details). In particular, for each positive integer $m$, let $H^{m}(\mathcal{O})$ denote the standard Sobolev spaces over the domain $\mathcal{O} \subset \mathbb{R}^{2}$ with norm $\|\cdot\|_{m, \mathcal{O}}$ and seminorm $|\cdot|_{m, \mathcal{O}}$. As usual $H_{0}^{m}(\mathcal{O})$ denotes the space of functions in $H^{m}(\mathcal{O})$ with vanishing trace. In addition, $(\cdot, \cdot)_{\mathcal{O}}$ will denote the $L^{2}(\mathcal{O})$ inner product. Conventionally the subscript will be omitted when $\mathcal{O}$ is computational domain $\Omega$.

For a Banach space $\mathcal{X}$ and $0<T_{0}<\infty, C^{0}\left(\left[0, T_{0}\right] ; \mathcal{X}\right)$ denotes the set of functions $f:\left[0, T_{0}\right] \rightarrow \mathcal{X}$ that are continuous in $t \in\left[0, T_{0}\right]$. For an integer $m \geq 1$ we define $C^{m}\left(\left[0, T_{0}\right] ; \mathcal{X}\right)=\left\{f \mid \partial^{l} f / \partial t^{l} \in C^{0}\left(\left[0, T_{0}\right] ; \mathcal{X}\right), 0 \leq l \leq m\right\}$, where $\partial^{l} f / \partial t^{l}$ is the l-st time derivative. For a function $f:\left[0, T_{0}\right] \rightarrow \mathcal{X}$, we define the space time norm

$$
\|f\|_{L^{p}\left(\left[0, T_{0}\right] ; \mathcal{X}\right)}= \begin{cases}\left(\int_{a}^{b}\|f\|_{\mathcal{X}}^{p} d t\right)^{1 / p}, & 1 \leq p<\infty \\ \operatorname{esssup}_{t \in\left[0, T_{0}\right]}\|f\|_{\mathcal{X}}, & p=\infty\end{cases}
$$

If the time interval is fixed as $\left[0, T_{0}\right]$, then we may write $L^{p} \mathcal{X}$ instead of $L^{p}\left(\left[0, T_{0}\right] ; \mathcal{X}\right)$. Also we denote by $W^{m, p}\left(\left[0, T_{0}\right] ; \mathcal{X}\right)$ the space of functions $f:\left[0, T_{0}\right] \rightarrow \mathcal{X}$ for which $\|f\|_{W^{m, p}\left(\left[0, T_{0}\right] ; \mathcal{X}\right)}<\infty$, where

$$
\|f\|_{W^{m, p}\left(\left[0, T_{0}\right] ; \mathcal{X}\right)}=\left\{\begin{array}{l}
\left(\sum_{l=0}^{m}\left\|\partial^{l} f / \partial t^{l}\right\|_{L^{p}\left(\left[0, T_{0}\right] ; \mathcal{X}\right)}^{p}\right)^{1 / p}, \quad 1 \leq p<\infty, \\
\max _{0 \leq l \leq m}\left\|\partial^{l} f / \partial t^{l}\right\|_{L^{\infty}\left(\left[0, T_{0}\right] ; \mathcal{X}\right)}, \quad p=\infty
\end{array}\right.
$$

For simplicity, we will use $\dot{f}, \ddot{f}, \ldots$, to denote $\partial f / \partial t, \partial^{2} f / \partial t^{2}, \ldots$, respectively.

Throughout the paper, if not particularly indicated, $C$ with or without subscripts, bars, tildes, or hats, will denote a generic positive constant independent of the mesh size $h$ and the Lamé coefficient $\lambda$.

\section{Biot's CONSOLIDATION MODEL}

Let $\Omega \subset \mathbb{R}^{2}$ be a polygonal domain. Throughout this paper, we are interested in the quasi-static Biot's consolidation model in poroelasticity. The governing equations of the model are

$$
\begin{aligned}
-\operatorname{div}(\mathcal{C} \varepsilon(\mathbf{u}))+\alpha \nabla p & =\mathbf{f}, \\
c_{0} \dot{p}+\alpha \operatorname{div} \dot{\mathbf{u}}-\operatorname{div}(\mathbf{K} \nabla p) & =g,
\end{aligned}
$$

where $\mathcal{C}$ is the elasticity stiffness tensor, $c_{0} \geq 0$ is the constrained specific storage coefficient, $\mathbf{K}$ is the hydraulic conductivity tensor, $\alpha>0$ is the Biot-Willis constant which is close to $1, \mathbf{f}$ is the body force and $g$ is the source/sink density function of the fluid. The primary unknowns are the fluid pressure $p$ and the displacement of the porous medium $\mathbf{u}$.

For isotropic elastic porous media, $\mathcal{C}$ has the form

$$
\mathcal{C} \boldsymbol{\tau}=2 \mu \boldsymbol{\tau}+\lambda \operatorname{tr}(\boldsymbol{\tau}) \mathbf{I}
$$


for all symmetric tensor $\boldsymbol{\tau}$, where the constant $\mu, \lambda>0$ are the Lamé constants, and $\mathbf{I}$ is the identity matrix. The hydraulic conductivity tensor $\mathbf{K}$ is a symmetric and uniform positive definite tensor satisfying: there exist positive constants $k_{\min }$ and $k_{\max }$ such that for any $x \in \Omega$

$$
k_{\min } \xi^{T} \xi \leq \xi^{T} \mathbf{K}(x) \xi \leq k_{\max } \xi^{T} \xi \quad \forall \xi \in \mathbb{R}^{2} .
$$

In order to complete the equations, we need suitable boundary and initial conditions. To this end, we assume that there are two independent partitions of $\partial \Omega$,

$$
\partial \Omega=\Gamma_{p} \cup \Gamma_{f}, \quad \partial \Omega=\Gamma_{d} \cup \Gamma_{t},
$$

with $\left|\Gamma_{p}\right|,\left|\Gamma_{d}\right|>0$. On the boundary $\partial \Omega$, we prescribe the following boundary conditions:

$$
\begin{aligned}
& p=0 \quad \text { on } \quad \Gamma_{p}, \quad \mathbf{w} \cdot \mathbf{n}=0 \quad \text { on } \quad \Gamma_{f}, \\
& \mathbf{u}=\mathbf{0} \quad \text { on } \Gamma_{d}, \quad \boldsymbol{\sigma} \cdot \mathbf{n}=\mathbf{0} \quad \text { on } \Gamma_{t},
\end{aligned}
$$

where $\mathbf{n}$ is the outward unit normal vector, $\mathbf{w}:=-\mathbf{K} \nabla p$ and $\boldsymbol{\sigma}:=\mathcal{C} \varepsilon(\mathbf{u})-\alpha p \mathbf{I}$. Here we only consider homogeneous boundary conditions for simplicity, but our method can be extended to nonhomogenous case without any difficulty. We also prescribe the following initial conditions:

$$
p(0)=p^{0} \quad \text { and } \quad \mathbf{u}(0)=\mathbf{u}^{0} \quad \text { in } \quad \Omega
$$

with $p^{0}=0$ on $\Gamma_{p}$ and $\mathbf{u}^{0}=\mathbf{0}$ on $\Gamma_{d}$. According to [62], we have the following regularity result.

Theorem 2.1. Let $(\mathbf{u}, p)$ be the solution of the Biot' model (2.1). Then, for $T>0$,

$$
\begin{aligned}
\sup _{0 \leq t \leq T}\|\mathbf{u}(t)\|_{2}+\lambda \sup _{0 \leq t \leq T}\|\operatorname{div} \mathbf{u}(t)\|_{1} \leq & C\left(\left\|p^{0}\right\|_{1}+\sup _{0 \leq t \leq T}\|\mathbf{f}(t)\|_{0}+\sup _{0 \leq t \leq T}\|g(t)\|_{0}\right. \\
& \left.+\left(\int_{0}^{T}\|\mathbf{f}(s)\|_{-1}^{2} \mathrm{~d} s\right)^{\frac{1}{2}}+\left(\int_{0}^{T}\|g(s)\|_{0}^{2} \mathrm{~d} s\right)^{\frac{1}{2}}\right),
\end{aligned}
$$

where $C=C\left(\omega, \Gamma_{d}, \mathbf{K}, \mu\right)$.

Remark 2.2. According to Remark 3.4. of [62], if the time derivatives of solution and data functions are smooth enough, the above inequality also holds for $\mathbf{u}_{t}$ and $\mathbf{u}_{t t}$.

\subsection{Variational formulation}

This section presents a three-field mixed variational formulation for the Biot's model. To this end, introducing a new unknown $\mathbf{w}=-\mathbf{K} \nabla p$ (called volumetric fluid flux), we have

$$
\begin{aligned}
-\operatorname{div}(\mathcal{C} \varepsilon(\mathbf{u}))+\alpha \nabla p & =\mathbf{f}, \\
\mathbf{K}^{-1} \mathbf{w}+\nabla p & =0, \\
c_{0} \dot{p}+\alpha \operatorname{div} \dot{\mathbf{u}}+\operatorname{div} \mathbf{w} & =g .
\end{aligned}
$$

Let

$$
\begin{aligned}
& \boldsymbol{V}:=\left\{\mathbf{v} \in\left[H^{1}(\Omega)\right]^{2}:\left.\mathbf{v}\right|_{\Gamma_{d}}=0\right\} \\
& \mathbf{\Sigma}:=\left\{\mathbf{z} \in H(\operatorname{div} ; \Omega):\left.\mathbf{z} \cdot \mathbf{n}\right|_{\Gamma_{f}}=0\right\} \\
& Q:=L^{2}(\Omega)
\end{aligned}
$$


and define bilinear forms

$$
\begin{aligned}
a(\mathbf{u}, \mathbf{v}) & :=2 \mu \tilde{a}(\mathbf{u}, \mathbf{v})+(\lambda \operatorname{div} \mathbf{u}, \operatorname{div} \mathbf{v}), \\
\tilde{a}(\mathbf{u}, \mathbf{v}) & :=(\varepsilon(\mathbf{u}), \varepsilon(\mathbf{v})), \\
m(\mathbf{w}, \mathbf{z}) & :=\left(\mathbf{K}^{-1} \mathbf{w}, \mathbf{z}\right) .
\end{aligned}
$$

Then, the mixed formulation of $(2.3)-(2.5)$ is to find $(\mathbf{u}, p) \in C^{1}\left(\left[0, T_{0}\right] ; \boldsymbol{V} \times Q\right)$ and $\mathbf{w} \in C^{0}\left(\left[0, T_{0}\right] ; \boldsymbol{\Sigma}\right)$ such that

$$
\begin{aligned}
a(\mathbf{u}, \mathbf{v})-\alpha(\operatorname{div} \mathbf{v}, p) & =(\mathbf{f}, \mathbf{v}), & & \forall \mathbf{v} \in \boldsymbol{V}, \\
m(\mathbf{w}, \mathbf{z})-(\operatorname{div} \mathbf{z}, p) & =0, & & \forall \mathbf{z} \in \mathbf{\Sigma}, \\
c_{0}(\dot{p}, q)+\alpha(\operatorname{div} \dot{\mathbf{u}}, q)+(\operatorname{div} \mathbf{w}, q) & =(g, q), & & \forall q \in Q .
\end{aligned}
$$

\section{VEM APPROXIMATION}

The main purpose of this section is to introduce some virtual element subspaces and discrete bilinear forms that are crucial in constructing a mixed virtual element method that can overcome Poisson locking when $\lambda \rightarrow \infty$ or pressure oscillations when $c_{0}=0$ and $\mathbf{K} \approx 0$. The key points to obtain such methods is to construct virtual element subspaces satisfying inf-sup conditions. Its a priori error estimates will be discussed in the next section. From now on, we only consider $\Gamma_{p}=\Gamma_{d}=\partial \Omega$ for simplicity. It is possible to expand our results to more general situation, but related proof is quite involved.

\subsection{Basic assumptions on mesh}

Let $\left\{\mathcal{T}_{h}\right\}_{h}$ be a sequence of decompositions of $\Omega$ into elements $E$, and let $\mathcal{E}_{h}$ be the sets of edges $e$ of $\mathcal{T}_{h}$. For each element $E \in \mathcal{T}_{h}, h_{E}$ denote its diameter. As usual, $h$ denote the maximum of the diameters of the elements in $\mathcal{T}_{h}$. We make the following mesh regularity assumptions which are standard in the context of VEM $[9,15,25,28,31]$.

Assumption 3.1. There exist constants $\rho_{1}, \rho_{2}>0$ such that

(1) every element $E$ is star-shaped with respect to a ball of radius $\geq \rho_{1} h_{E}$,

(2) the distance between any two vertices of $E$ is $\geq \rho_{2} h_{E}$.

Remark 3.2. An immediate consequence of the above assumptions is that each element $E$ admits a subtriangulation $\mathcal{T}_{h}^{E}$ whose union $\widetilde{\mathcal{T}}_{h}:=\cup_{E \in \mathcal{T}_{h}} \mathcal{T}_{h}^{E}$ is a shape regular triangulation.

Remark 3.3. According to [25], the above assumptions also admit the following scaled trace inequality

$$
h_{E}^{-1}\|v\|_{0, \partial E}^{2} \leq C\left(h_{E}^{-2}\|v\|_{0, E}^{2}+|v|_{1, E}^{2}\right) \quad \forall v \in H^{1}(E)
$$

with $C>0$ independent of $E$.

\subsection{Virtual element subspaces}

This subsection devotes to the introduction of two conforming virtual elements subspaces $\boldsymbol{V}_{h} \subset \boldsymbol{V}$ and $\boldsymbol{\Sigma}_{h} \subset \boldsymbol{\Sigma}$.

\subsection{1. $H^{1}$-conforming virtual element subspaces}

We start with the $H^{1}$-conforming virtual element subspaces discussed in [2]. For this purpose, we recall the following spaces: for any $k \in \mathbb{N}$ and $E \in \mathcal{T}_{h}$,

- $\mathbb{P}_{k}(E)$ the set of polynomials of degree $\leq k$ on $E$ (with extended notation $\mathbb{P}_{-1}(E)=\{0\}$ ),

- $\mathbb{B}_{k}(\partial E):=\left\{v \in C^{0}(\partial E):\left.v\right|_{e} \in \mathbb{P}_{k}(e)\right.$ for each edge $e$ of $\left.\partial E\right\}$,

- $\widetilde{V}_{k}^{E}:=\left\{v_{h} \in H^{1}(E) \cap C^{0}(E): \Delta v_{h} \in \mathbb{P}_{k}(E),\left.v_{h}\right|_{\partial E} \in \mathbb{B}_{k}(\partial E)\right\}$. 
If $\mathcal{O}$ is a subset of $\mathbb{R}^{2}$, we denote by $\mathbf{x}_{\mathcal{O}}, h_{\mathcal{O}}$ and $|\mathcal{O}|$ the centroid, the diameter and the measure of $\mathcal{O}$, respectively. For $r \in \mathbb{N}$, we denote by $\mathcal{M}_{r}(\mathcal{O})$ the set of scaled monomials

$$
\mathcal{M}_{r}(\mathcal{O}):=\left\{m: m=\left(\frac{\mathbf{x}-\mathbf{x}_{\mathcal{O}}}{h_{\mathcal{O}}}\right)^{\mathbf{s}} \text { for } \quad \mathbf{s} \in \mathbb{N}^{2} \quad \text { with } \quad|\mathbf{s}| \leq r\right\},
$$

where $\mathbf{s}=\left(s_{1}, s_{2}\right),|\mathbf{s}|=s_{1}+s_{2}$ and $\mathbf{x}^{\mathbf{s}}=x_{1}^{s_{1}} x_{2}^{s_{2}}$. We also need the following set defined by

$$
\mathcal{M}_{r}^{*}(\mathcal{O}):=\left\{m: m=\left(\frac{\mathbf{x}-\mathbf{x}_{\mathcal{O}}}{h_{\mathcal{O}}}\right)^{\mathbf{s}} \text { for } \quad \mathbf{s} \in \mathbb{N}^{2} \quad \text { with } \quad|\mathbf{s}|=r\right\} .
$$

Then for any $k \in \mathbb{N}$ and $E \in \mathcal{T}_{h}$, we define the following useful polynomial projections:

- the $L^{2}$-projection for scalar functions $\Pi_{k}^{0, E}: \widetilde{V}_{k}^{E} \rightarrow \mathbb{P}_{k}(E)$, given by

$$
\int_{E} q_{k}\left(v-\Pi_{k}^{0, E} v\right) \mathrm{d} E=0 \quad \forall v \in L^{2}(E) \quad \text { and } \quad \forall q_{k} \in \mathbb{P}_{k}(E),
$$

- the $H^{1}$ semi-norm projection for scalar functions $\Pi_{k}^{\nabla, E}: \widetilde{V}_{k}^{E} \rightarrow \mathbb{P}_{k}(E)$, defined by

$$
\begin{cases}\int_{E} \nabla q_{k} \nabla\left(v-\Pi_{k}^{\nabla, E} v\right) \mathrm{d} E=0 & \forall v \in H^{1}(E) \quad \text { and } \quad \forall q_{k} \in \mathbb{P}_{k}(E), \\ \int_{\partial E}\left(v-\Pi_{k}^{\nabla, E} v\right) \mathrm{d} s=0 & \text { if } \quad k=1, \\ \int_{E}\left(v-\Pi_{k}^{\nabla, E} v\right) \mathrm{d} E=0 & \text { if } \quad k \geq 2 .\end{cases}
$$

For simplicity, we still use $\Pi_{k}^{0, E}$ and $\Pi_{k}^{\nabla, E}$ for their extension to vector and tensor functions. Set $\mathbb{P}_{k}\left(\mathcal{T}_{h}\right)=\{p \in$ $\left.L^{2}(\Omega):\left.p\right|_{E} \in \mathbb{P}_{k}(E) \forall E \in \mathcal{T}_{h}\right\}$. We denote the global version of $\Pi_{k}^{0, E}$ by $\Pi_{k}^{0}: L^{2}(\Omega) \rightarrow \mathbb{P}_{k}\left(\mathcal{T}_{h}\right)$, which is given by

$$
\left.\left(\Pi_{k}^{0} \mathbf{v}\right)\right|_{E}:=\Pi_{k}^{0, E}\left(\left.\mathbf{v}\right|_{E}\right), \quad \forall E \in \mathcal{T}_{h}, \quad \forall \mathbf{v} \in L^{2}(\Omega) .
$$

The $L^{2}$ projection defined in (3.2) satisfies the following approximation property. Details can be found in [24,25].

Theorem 3.4. Suppose that Assumption 3.1 is satisfied. Then, for any $w \in H^{m}(E)$, with $1 \leq m \leq k+1$, it holds

$$
\left\|w-\Pi_{k}^{0, E} w\right\|_{0, E}+h_{E}\left|w-\Pi_{k}^{0, E} w\right|_{1, E} \leq C h_{E}^{m}|w|_{m, E} .
$$

The positive constant $C$ depends only on the polynomial degree $k$ and the mesh regularity.

We now recall the local virtual element space of order $k$ (see [2] for more detail): $\forall E \in \mathcal{T}_{h}$

$$
V_{k}^{E}:=\left\{v_{h} \in \widetilde{V}_{k}^{E}:\left(q_{h}, v_{h}\right)_{E}=\left(q_{h}, \Pi_{k}^{\nabla, E} v_{h}\right)_{E} \quad \forall q_{h}^{*} \in \mathcal{M}_{k-1}^{*}(E) \cup \mathcal{M}_{k}^{*}(E)\right\},
$$

Following [2], the degrees of freedom guaranteeing unisolvency for each $v \in V_{k}^{E}$ are defined by

- $\widetilde{\mathbf{D}}_{\mathbf{V}} \mathbf{1}$ : the values of $v$ at the vertices of the polygon $E$,

- $\widetilde{\mathbf{D}}_{\mathbf{V}}$ 2: the moments of $v$ on edges up to degree $k-2$,

$$
|e|^{-1} \int_{e} q v \mathrm{~d} s \quad \forall q \in \mathcal{M}_{k-2}(e) \quad \forall \text { edge } e,
$$

- $\widetilde{\mathbf{D}}_{\mathbf{V}} \mathbf{3}$ : the moments of $v$ on element $E$ up to degree $k-2$,

$$
|E|^{-1} \int_{E} q v \mathrm{~d} E \quad \forall q \in \mathcal{M}_{k-2}(E) .
$$


Remark 3.5. It is worth pointing out that $\Pi_{k}^{0, E}$ and $\Pi_{k}^{\nabla, E}$ are computable from the knowledge of the degrees of freedom $\widetilde{\mathbf{D}}_{\mathbf{V}} \mathbf{1}-\widetilde{\mathbf{D}}_{\mathbf{V}} \mathbf{3}$. This fact was proved in [2].

The global virtual element space $V_{k}$ can be defined by

$$
V_{k}=\left\{v \in H^{1}(\Omega):\left.v\right|_{E} \in V_{k}^{E}\right\} .
$$

Now we set

$$
\boldsymbol{V}_{h}:=\left[V_{k}\right]^{2} \cap \boldsymbol{V} \quad k \geq 1 .
$$

The proof of the following lemma is quite similar to the one given in the reference [8] and so is omitted. We remark that the following operator is essentially the one of [8].

Lemma 3.6. If Assumption 3.1 holds and $k \geq 2$, then there exists a operator $\Pi_{F}: \boldsymbol{V} \rightarrow \boldsymbol{V}_{h}$ satisfying

$$
\begin{aligned}
\left(\operatorname{div}\left(\mathbf{v}-\Pi_{F} \mathbf{v}\right), q_{h}\right) & =0 \quad \forall \mathbf{v} \in\left[H_{0}^{1}(\Omega)\right]^{2} \quad \forall q_{h} \in \mathbb{P}_{k-1}\left(\mathcal{T}_{h}\right) \cap L_{0}^{2}(\Omega), \\
\left|\Pi_{F} \mathbf{v}\right|_{1} & \leq C|\mathbf{v}|_{1} .
\end{aligned}
$$

In addition, if $\mathbf{v} \in\left[H^{s}(\Omega)\right]^{2}, 1 \leq s \leq k+1$, then we have

$$
\left|\mathbf{v}-\Pi_{F} \mathbf{v}\right|_{m} \leq C h_{E}^{s-m}\|\mathbf{v}\|_{s},
$$

for $m=0,1$ with a positive constant $C$ independent of $h$ and $\mathbf{v}$.

Using Lemma 3.6, we can establish the following inf-sup condition.

Theorem 3.7. Under the same assumptions of Lemma 3.6, the following inf-sup condition holds

$$
\inf _{q_{h} \in \mathbb{P}_{k-1}\left(\mathcal{T}_{h}\right) \cap L_{0}^{2}(\Omega),} \sup _{\substack{q_{h} \neq 0 \\ \mathbf{v}_{h} \in V_{h}, \mathbf{v}_{h} \neq 0}} \frac{\left(\operatorname{div} \mathbf{v}_{h}, q_{h}\right)}{\left|\mathbf{v}_{h}\right|_{1}\left\|q_{h}\right\|_{0}} \geq C,
$$

with $C>0$ independent of $h$.

\subsection{2. $H(\mathrm{div})$-conforming virtual element subspaces}

We now turn to the $H\left(\right.$ div)-conforming virtual element subspace given in [14]. For every $E \in \mathcal{T}_{h}$ and integer $k \geq 0$, we introduce:

$$
\mathcal{G}_{k}(E):=\nabla \mathbb{P}_{k+1}(E)
$$

and

$$
\mathcal{G}_{k}^{\perp}(E):=\text { the } L^{2}(E) \text { orthogonal of } \mathcal{G}_{k}(E) \text { in }\left[\mathbb{P}_{k}(E)\right]^{2} \text {. }
$$

Clearly, we have

$$
\left[\mathbb{P}_{k}(E)\right]^{2}=\mathcal{G}_{k}(E) \oplus \mathcal{G}_{k}^{\perp}(E) .
$$

For integer $k \geq 0$, we define

$$
\begin{aligned}
\Sigma_{h}^{k}(E):= & \left\{\mathbf{z} \in H(\operatorname{div} ; E) \cap H(\operatorname{curl} ; E): \mathbf{z} \cdot \mathbf{n} \in \mathbb{P}_{k}(e) \text { for each edge } e \text { of } \partial E,\right. \\
& \left.\operatorname{divz} \in \mathbb{P}_{k}(E), \text { and } \operatorname{curl} \mathbf{z} \in \mathbb{P}_{k-1}(E)\right\} .
\end{aligned}
$$

Then we set

$$
\Sigma_{h}^{k}:=\left\{\mathbf{z} \in H(\operatorname{div} ; \Omega) \text { such that }\left.\mathbf{z}\right|_{E} \in \Sigma_{h}^{k}(E) \quad \forall E \in \mathcal{T}_{h}\right\}
$$


The degrees of freedom for $\Sigma_{h}^{k}$ are defined by

$$
\begin{array}{ll}
|e|^{-1} \int_{e} \mathbf{z} \cdot \mathbf{n} q_{k} \mathrm{~d} s & \text { for all edge } e, \text { for all } q_{k} \in \mathbb{P}_{k}(e), \\
|E|^{-1} \int_{E} \mathbf{z} \cdot \mathbf{g}_{k-1} \mathrm{~d} x & \text { for all element } E, \text { for all } \mathbf{g}_{k-1} \in \mathcal{G}_{k-1}(E), \\
|E|^{-1} \int_{E} \mathbf{z} \cdot \mathbf{g}_{k}^{\perp} \mathrm{d} x & \text { for all element } E, \text { for all } \mathbf{g}_{k}^{\perp} \in \mathcal{G}_{k}^{\perp}(E) .
\end{array}
$$

According to [14], the degrees of freedom (3.11)-(3.13) can guarantee the unisolvency for each $\boldsymbol{\tau} \in \Sigma_{h}^{k}$.

We also need interpolation operator $\boldsymbol{\Pi}_{k}^{i}:\left[H^{1}(\Omega)\right]^{2} \rightarrow \Sigma_{h}^{k}$ defined by

$$
\begin{array}{ll}
\int_{e}\left(\mathbf{z}-\boldsymbol{\Pi}_{k}^{i} \mathbf{z}\right) \cdot \mathbf{n} q_{k} \mathrm{~d} s=0 & \text { for all edge } e, \text { for all } q_{k} \in \mathbb{P}_{k}(e), \\
\int_{E}\left(\mathbf{z}-\boldsymbol{\Pi}_{k}^{i} \mathbf{z}\right) \cdot \mathbf{g}_{k-1} \mathrm{~d} x=0 & \text { for all element } E, \text { for all } \mathbf{g}_{k-1} \in \mathcal{G}_{k-1}(E), \\
\int_{E}\left(\mathbf{z}-\boldsymbol{\Pi}_{k}^{i} \mathbf{z}\right) \cdot \mathbf{g}_{k}^{\perp} \mathrm{d} x=0 & \text { for all element } E, \text { for all } \mathbf{g}_{k}^{\perp} \in \mathcal{G}_{k}^{\perp}(E) .
\end{array}
$$

From [14] we have

$$
\operatorname{div} \Pi_{k}^{i} \mathbf{z}=\Pi_{k}^{0} \operatorname{div} \mathbf{z}
$$

Moreover, the following estimates hold, provided $\mathbf{z}$ is smooth enough:

$$
\begin{gathered}
\left\|\mathbf{z}-\boldsymbol{\Pi}_{k}^{i} \mathbf{z}\right\|_{0, E} \leq C h^{s}|\mathbf{z}|_{s, E} 0 \leq s \leq k+1, \\
\left\|\operatorname{div} \mathbf{z}-\operatorname{div} \boldsymbol{\Pi}_{k}^{i} \mathbf{z}\right\|_{0, E} \leq C h^{s}|\operatorname{div} \mathbf{z}|_{s, E} 0 \leq s \leq k+1 .
\end{gathered}
$$

Let

$$
\boldsymbol{\Sigma}_{h}:=\Sigma_{h}^{k-1} \cap \boldsymbol{\Sigma}, \quad Q_{h}:=\mathbb{P}_{k-1}\left(\mathcal{T}_{h}\right), \quad k \geq 1 .
$$

Using (3.14), we can get the following inf-sup condition.

Theorem 3.8. There exists a positive constant $C$ independent of $h$ such that

$$
\inf _{\substack{q_{h} \in Q_{h}, q_{h} \neq 0}} \sup _{\substack{\mathbf{z}_{h} \in \Sigma_{h} \\ \mathbf{z}_{h} \neq 0}} \frac{\left(\operatorname{div}_{h}, q_{h}\right)}{\left\|\mathbf{z}_{h}\right\|_{\operatorname{div}}\left\|q_{h}\right\|_{0}} \geq C .
$$

\subsection{Discrete bilinear forms and load term approximation}

The next step in the construction of our method is to define a discrete version of the bilinear forms $a(\cdot, \cdot)$, $\tilde{a}(\cdot, \cdot)$ and $m(\cdot, \cdot)$ defined in $(2.6)$. Obviously, we can split the following bilinear forms as:

$$
\begin{aligned}
a(\mathbf{u}, \mathbf{v}) & :=\sum_{E \in \mathcal{T}_{h}} a^{E}(\mathbf{u}, \mathbf{v}):=\sum_{E \in \mathcal{T}_{h}} 2 \mu \tilde{a}^{E}(\mathbf{u}, \mathbf{v})+\lambda(\operatorname{div} \mathbf{u}, \operatorname{div} \mathbf{v})_{E}, \\
m(\mathbf{w}, \mathbf{z}) & :=\sum_{E \in \mathcal{T}_{h}} m^{E}(\mathbf{w}, \mathbf{z}):=\sum_{E \in \mathcal{T}_{h}} m^{E}(\mathbf{w}, \mathbf{z})
\end{aligned}
$$

where

$$
\begin{aligned}
\tilde{a}^{E}(\mathbf{u}, \mathbf{v}) & :=(\varepsilon(\mathbf{u}), \boldsymbol{\varepsilon}(\mathbf{v}))_{E}, \\
m^{E}(\mathbf{w}, \mathbf{z}) & :=\left(\mathbf{K}^{-1} \mathbf{w}, \mathbf{z}\right)_{E} .
\end{aligned}
$$


We start with the construction of $a_{h}(\cdot, \cdot)$, then $m_{h}(\cdot, \cdot)$ can be constructed similarly. It is easy to see that for all $\mathbf{u}, \mathbf{v} \in\left[V_{k}^{E}\right]^{2}$, the quantity $a^{E}(\mathbf{u}, \mathbf{v})$ is not computable. Therefore, we need to define a computable discrete local bilinear form $a_{h}^{E}(\cdot, \cdot)$ which can approximate the continuous bilinear form $a^{E}(\cdot, \cdot)$. More precisely, $a_{h}^{E}(\cdot, \cdot)$ is defined by: for all $\mathbf{u}, \mathbf{v} \in\left[V_{k}^{E}\right]^{2}$

$$
a_{h}^{E}(\mathbf{u}, \mathbf{v}):=2 \mu \tilde{a}_{h}^{E}(\mathbf{u}, \mathbf{v})+\lambda\left(\Pi_{k-1}^{0, E} \operatorname{div} \mathbf{u}, \Pi_{k-1}^{0, E} \operatorname{div} \mathbf{v}\right)_{E},
$$

where

$$
\tilde{a}_{h}^{E}(\mathbf{u}, \mathbf{v}):=\left(\Pi_{k-1}^{0, E} \varepsilon(\mathbf{u}), \Pi_{k-1}^{0, E} \varepsilon(\mathbf{v})\right)_{E}+S^{E}(\mathbf{u}, \mathbf{v})
$$

and $S^{E}(\mathbf{u}, \mathbf{v})$ is a stabilizing bilinear form to be defined later. Clearly, for each $\mathbf{v} \in\left[V_{k}^{E}\right]^{2}$, we can write it as $\mathbf{v}=\left(v_{1}, v_{2}\right)$. Then the stabilizing term $S^{E}$ can be defined by

$$
S^{E}(\mathbf{u}, \mathbf{v}):=\sum_{j=1}^{2} \sum_{i=1}^{n_{k}^{E}} \chi_{i}^{k}\left(u_{j}-\Pi_{k}^{\nabla, E} u_{j}\right) \chi_{i}^{k}\left(v_{j}-\Pi_{k}^{\nabla, E} v_{j}\right)
$$

where $n_{k}^{E}$ is the dimension of $V_{k}^{E}$, and $\chi_{i}^{k}\left(i \in\left\{1,2, \cdots, n_{k}^{E}\right\}\right)$ are the degrees of freedom given by $\widetilde{\mathbf{D}}_{\mathbf{V}} \mathbf{1}-\widetilde{\mathbf{D}}_{\mathbf{V}} \mathbf{3}$.

We define the global approximated bilinear form $a_{h}(\cdot, \cdot): \boldsymbol{V}_{h} \times \boldsymbol{V}_{h} \rightarrow \mathbb{R}$ by simply summing the local bilinear forms:

$$
a_{h}(\mathbf{u}, \mathbf{v}):=\sum_{E \in \mathcal{T}_{h}} a_{h}^{E}(\mathbf{u}, \mathbf{v}) \quad \forall \mathbf{u}, \mathbf{v} \in \boldsymbol{V}_{h} .
$$

Following standard techniques in the literature [13] we can easily get the following lemma.

Lemma 3.9. For $k \geq 1$, the stabilizing term defined in (3.20) satisfies

- -consistency: for all $\mathbf{q} \in\left[\mathbb{P}_{k}(E)\right]^{2}$ and $\mathbf{v} \in\left[V_{k}^{E}\right]^{2}$

$$
S^{E}(\mathbf{q}, \mathbf{v})=0,
$$

- stability:

$$
\beta_{*}\left\|\left(I-\Pi_{k-1}^{0, E}\right) \varepsilon(\mathbf{v})\right\|_{0, E}^{2} \leq S^{E}(\mathbf{v}, \mathbf{v}) \leq \beta^{*}\left\|\nabla\left(\mathbf{v}-\Pi_{k}^{\nabla, E} \mathbf{v}\right)\right\|_{0, E}^{2} \quad \forall \mathbf{v} \in\left[V_{k}^{E}\right]^{2}
$$

with positive constants $\beta_{*}$ and $\beta^{*}$ independent of the element $E$.

Using the above lemma, we can easily get following lemma:

Lemma 3.10. The local discrete bilinear form $\tilde{a}_{h}^{E}(\cdot, \cdot)$ defined by (3.19) satisfies

- -consistency: for all $\mathbf{q} \in\left[\mathbb{P}_{k}(E)\right]^{2}$ and $\mathbf{v} \in\left[V_{k}^{E}\right]^{2}$

$$
\tilde{a}_{h}^{E}(\mathbf{q}, \mathbf{v})=\tilde{a}^{E}(\mathbf{q}, \mathbf{v})
$$

- stability:

$$
\beta_{*}\|\varepsilon(\mathbf{v})\|_{0, E}^{2} \leq \tilde{a}_{h}^{E}(\mathbf{v}, \mathbf{v}) \leq \beta^{*}\|\nabla \mathbf{v}\|_{0, E}^{2} \quad \forall \mathbf{v} \in\left[V_{k}^{E}\right]^{2}
$$

with positive constants $\beta_{*}$ and $\beta^{*}$ independent of the element $E$.

By Lemmas 3.9 and 3.10, we have following theorem.

Theorem 3.11. The discrete bilinear form $a_{h}(\cdot, \cdot): \boldsymbol{V}_{h} \times \boldsymbol{V}_{h} \rightarrow \mathbb{R}$ defined by (3.21) satisfies 
- boundedness: for all $\mathbf{u}, \mathbf{v} \in \boldsymbol{V}_{h}$

$$
a_{h}(\mathbf{u}, \mathbf{v}) \leq C_{\lambda}|\mathbf{u}|_{1}|\mathbf{v}|_{1},
$$

with $C_{\lambda}>0$ dependent on $\lambda$

- coercivity: for all $\mathbf{v} \in \boldsymbol{V}_{h}$

$$
a_{h}(\mathbf{v}, \mathbf{v}) \geq C|\mathbf{v}|_{1}^{2}
$$

with $C>0$ independent of $h$.

We now turn to the construction of $m_{h}(\cdot, \cdot)$. As $a_{h}(\cdot, \cdot), m_{h}(\cdot, \cdot)$ is defined element by element:

$$
m_{h}(\mathbf{w}, \mathbf{z}):=\sum_{E \in \mathcal{T}_{h}} m_{h}^{E}(\mathbf{w}, \mathbf{z}) .
$$

For each element $E \in \mathcal{T}_{h}$, we define a local bilinear form $m_{h}^{E}: \Sigma_{h}^{k}(E) \times \Sigma_{h}^{k}(E) \rightarrow R$ as

$$
m_{h}^{E}\left(\mathbf{w}_{h}, \mathbf{z}_{h}\right):=\left(\mathbf{K}^{-1} \Pi_{k}^{0, E} \mathbf{w}_{h}, \Pi_{k}^{0, E} \mathbf{z}_{h}\right)_{E}+S_{m}^{E}\left(\mathbf{w}_{h}-\Pi_{k}^{0, E} \mathbf{w}_{h}, \mathbf{z}_{h}-\Pi_{k}^{0, E} \mathbf{z}_{h}\right),
$$

where $S_{m}^{E}\left(\mathbf{w}_{h}-\Pi_{k}^{0, E} \mathbf{w}_{h}, \mathbf{z}_{h}-\Pi_{k}^{0, E} \mathbf{z}_{h}\right)$ is any symmetric and positive definite bilinear form such that

$$
\alpha_{*}\left\|\mathbf{z}_{h}-\Pi_{k}^{0, E} \mathbf{z}_{h}\right\|_{0, E}^{2} \leq \boldsymbol{S}_{h}^{E}\left(\mathbf{z}_{h}-\Pi_{k}^{0, E} \mathbf{z}_{h}, \mathbf{z}_{h}-\Pi_{k}^{0, E} \mathbf{z}_{h}\right) \leq \alpha^{*}\left\|\mathbf{z}_{h}-\Pi_{k}^{0, E} \mathbf{z}_{h}\right\|_{0, E}^{2},
$$

with $\alpha_{*}$ and $\alpha^{*}$ independent of $h$ and $E$. Such a bilinear form has been given in reference [14] and we just simply describe it. For each $E \in \mathcal{T}_{h}$, let $N_{E}$ be the number of local degrees of freedom (3.11)-(3.13), and denote by $\tilde{\chi}_{i}$, for $i=1,2, \ldots, N_{E}$, the operator that selects the i-th local degree of freedom (3.11)-(3.13). Then $S_{m}^{E}(\cdot, \cdot)$ is given by

$$
S_{m}^{E}\left(\mathbf{w}_{h}-\Pi_{k}^{0, E} \mathbf{w}_{h}, \mathbf{z}_{h}-\Pi_{k}^{0, E} \mathbf{z}_{h}\right):=|E| \sum_{i=1}^{N_{E}} \widetilde{\chi}_{i}\left(\mathbf{w}_{h}-\Pi_{k}^{0, E} \mathbf{w}_{h}\right) \widetilde{\chi}_{i}\left(\mathbf{z}_{h}-\Pi_{k}^{0, E} \mathbf{z}_{h}\right) \quad \forall \mathbf{w}_{h}, \mathbf{z}_{h} \in \Sigma_{h}^{k}(E) .
$$

It follows from (3.29) that

$$
\begin{aligned}
m_{h}^{E}\left(\mathbf{w}_{h}, \mathbf{z}_{h}\right) & \leq C\left\|\mathbf{w}_{h}\right\|_{0, E}\left\|\mathbf{z}_{h}\right\|_{0, E}, & & \forall \mathbf{w}_{h}, \mathbf{z}_{h} \in \Sigma_{h}^{k}(E), \\
C\left\|\mathbf{w}_{h}\right\|_{0, E}^{2} & \leq m_{h}^{E}\left(\mathbf{w}_{h}, \mathbf{w}_{h}\right) \leq C\left\|\mathbf{w}_{h}\right\|_{0, E}^{2} & & \forall \mathbf{w}_{h} \in \Sigma_{h}^{k}(E) .
\end{aligned}
$$

As a consequence, we have the following theorem for $m_{h}(\cdot, \cdot)$.

Theorem 3.12. The discrete bilinear form $m_{h}(\cdot, \cdot): \boldsymbol{\Sigma}_{h} \times \boldsymbol{\Sigma}_{h} \rightarrow \mathbb{R}$ defined by (3.28) satisfies

- boundedness: for all $\mathbf{w}, \mathbf{z} \in \boldsymbol{\Sigma}_{h}$

$$
m_{h}(\mathbf{w}, \mathbf{z}) \leq C\|\mathbf{w}\|_{\text {div }}\|\mathbf{z}\|_{\text {div }},
$$

with $C_{\lambda}>0$ dependent on $\lambda$

- coercivity: for all $\mathbf{z} \in \boldsymbol{Z}_{h}$

$$
m_{h}(\mathbf{z}, \mathbf{z}) \geq C\|\mathbf{z}\|_{\text {div }}^{2}
$$

with $C>0$ independent of $h$, where $\boldsymbol{Z}_{h}:=\left\{\mathbf{z} \in \boldsymbol{\Sigma}_{h}: \operatorname{div} \mathbf{z}=0\right\}$.

Finally we introduce how to approximate the load term $(\mathbf{f}, \mathbf{v})$, which, in general, is not computable. We define the approximated load term $\mathbf{f}_{h}$ as

$$
\left.\mathbf{f}_{h}\right|_{E}:=\Pi_{k}^{0, E} \mathbf{f} \quad \forall E \in \mathcal{T}_{h},
$$

and consider

$$
\left(\mathbf{f}_{h}, \mathbf{v}_{h}\right)=\sum_{E \in \mathcal{T}_{h}} \int_{E} \mathbf{f}_{h} \cdot \mathbf{v}_{h}:=\sum_{E \in \mathcal{T}_{h}} \int_{E} \Pi_{k}^{0, E} \mathbf{f} \cdot \mathbf{v}_{h}=\sum_{E \in \mathcal{T}_{h}} \int_{E} \mathbf{f} \cdot \Pi_{k}^{0, E} \mathbf{v}_{h} .
$$

According to [2,10], (3.34) can be exactly computed from $\widetilde{\mathbf{D}}_{\mathbf{V}} \mathbf{1}-\widetilde{\mathbf{D}}_{\mathbf{V}} \mathbf{3}$. 


\section{Fully Discrete SCHEME}

In this section, we shall deal with the a priori analysis of a fully discrete scheme of (2.3)-(2.5). Suppose that $T_{0}=N \Delta t$, where $N$ is a positive integer. Set $t_{n}=n \Delta t, n=0,1, \cdots, N$. For a continuous function $g$ defined on $\left[0, T_{0}\right]$, we define $g^{n}=g\left(t_{n}\right)$. Then, given initial data $\mathbf{u}_{h}^{0} \in \boldsymbol{V}_{h}, p_{h}^{0} \in Q_{h}$, the fully discrete scheme of (2.3)-(2.5) seeks $\left(\mathbf{u}_{h}^{n}, \mathbf{w}_{h}^{n}, p_{h}^{n}\right) \in \boldsymbol{V}_{h} \times \boldsymbol{\Sigma}_{h} \times Q_{h}$ at time $t_{n}, 1 \leq n \leq N$, such that

$$
\begin{aligned}
a_{h}\left(\mathbf{u}_{h}^{n}, \mathbf{v}_{h}\right)-\alpha\left(\operatorname{div}_{h}, p_{h}^{n}\right) & =\left(\mathbf{f}_{h}^{n}, \mathbf{v}_{h}\right), & & \forall \mathbf{v}_{h} \in \boldsymbol{V}_{h}, \\
m_{h}\left(\mathbf{w}_{h}^{n}, \mathbf{z}_{h}\right)-\left(\operatorname{div} \mathbf{z}_{h}, p_{h}^{n}\right) & =0, & & \forall \mathbf{z}_{h} \in \mathbf{\Sigma}_{h}, \\
c_{0}\left(\frac{p_{h}^{n}-p_{h}^{n-1}}{\Delta t}, q_{h}\right)+\alpha\left(\operatorname{div}\left(\frac{\mathbf{u}_{h}^{n}-\mathbf{u}_{h}^{n-1}}{\Delta t}\right), q_{h}\right)+\left(\operatorname{div}_{h}^{n}, q_{h}\right) & =\left(g^{n}, q_{h}\right), & & \forall q_{h} \in Q_{h} .
\end{aligned}
$$

It is easy to check that all the $L^{2}$ product terms are computable. This system can be rewritten as:

$$
\begin{aligned}
a_{h}\left(\mathbf{u}_{h}^{n}, \mathbf{v}_{h}\right)-\alpha\left(\operatorname{div}_{h}, p_{h}^{n}\right) & =\left(\mathbf{f}_{h}^{n}, \mathbf{v}_{h}\right), & & \forall \mathbf{v}_{h} \in \boldsymbol{V}_{h}, \\
\Delta t m_{h}\left(\mathbf{w}_{h}^{n}, \mathbf{z}_{h}\right)-\Delta t\left(\operatorname{div}_{h}, p_{h}^{n}\right) & =0, & & \forall \mathbf{z}_{h} \in \mathbf{\Sigma}_{h}, \\
c_{0}\left(p_{h}^{n}, q_{h}\right)+\alpha\left(\operatorname{div} \mathbf{u}_{h}^{n}, q_{h}\right)+\Delta t\left(\operatorname{div}_{h}^{n}, q_{h}\right) & =\Delta t\left(g^{n}, q_{h}\right)+c_{0}\left(p_{h}^{n-1}, q_{h}\right)+\alpha\left(\operatorname{div}_{h}^{n-1}, q_{h}\right), & & \forall q_{h} \in Q_{h} .
\end{aligned}
$$

Obviously, this is a square system of linear equations for the unknowns $\left(\mathbf{u}_{h}^{n}, \mathbf{w}_{h}^{n}, p_{h}^{n}\right)$. It suffices to prove its uniqueness. Assume that $\mathbf{u}_{h}^{n-1}, \mathbf{w}_{h}^{n-1}, p_{h}^{n-1}, \mathbf{f}^{n}$ and $g^{n}$ vanish. Setting $\mathbf{v}_{h}=\mathbf{u}_{h}^{n}, \mathbf{z}_{h}=\mathbf{w}_{h}^{n}$ and $q_{h}=p_{h}^{n}$ and adding them together leads to

$$
a_{h}\left(\mathbf{u}_{h}^{n}, \mathbf{u}_{h}^{n}\right)+\Delta t m_{h}\left(\mathbf{w}_{h}^{n}, \mathbf{w}_{h}^{n}\right)+c_{0}\left(p_{h}^{n}, p_{h}^{n}\right)=0 .
$$

From (3.27) and (3.31), we can get $\left\|\mathbf{u}_{h}^{n}\right\|_{1}=0,\left\|\mathbf{w}_{h}^{n}\right\|_{0}=0$ and $\left\|p_{h}^{n}\right\|_{0}=0$, for $c_{0}>0$. If $c_{0}=0$, we still have $\left\|\mathbf{u}_{h}^{n}\right\|_{1}=0,\left\|\mathbf{w}_{h}^{n}\right\|_{0}=0$, from which we infer

$$
\left(\operatorname{div} \mathbf{z}_{h}, p_{h}^{n}\right)=0, \quad \forall \mathbf{z}_{h} \in \mathbf{\Sigma}_{h} .
$$

Using Theorem 3.8, we have $\left\|p_{h}^{n}\right\|_{0}=0$. To conclude, we have established the following result.

Theorem 4.1. At each time step $t_{n}$, the fully discrete scheme (4.1)-(4.3) has a unique solution $\left(\mathbf{u}_{h}^{n}, \mathbf{w}_{h}^{n}, p_{h}^{n}\right) \in$ $V_{h} \times \Sigma_{h} \times Q_{h}$.

\subsection{Elliptic projection}

To define initial data $\mathbf{u}_{h}^{0} \in \boldsymbol{V}_{h}, p_{h}^{0} \in Q_{h}$ and derive the error analysis of the fully discrete scheme (4.1)-(4.3), we need a elliptic projection $\Pi_{e}: \boldsymbol{V} \times \boldsymbol{\Sigma} \times Q \rightarrow \boldsymbol{V}_{h} \times \boldsymbol{\Sigma}_{h} \times Q_{h}$ defined below. Given $(\mathbf{u}, \mathbf{w}, p) \in \boldsymbol{V} \times \boldsymbol{\Sigma} \times Q$, let $\left(\mathbf{u}_{\pi}, \mathbf{w}_{\pi}, p_{\pi}\right)=\Pi_{e}(\mathbf{u}, \mathbf{w}, p)$ be the solution of the following equations

$$
\begin{aligned}
a_{h}\left(\mathbf{u}_{\pi}, \mathbf{v}_{h}\right)-\alpha\left(\operatorname{div} \mathbf{v}_{h}, p_{\pi}\right) & =a\left(\mathbf{u}, \mathbf{v}_{h}\right)-\alpha\left(\operatorname{div} \mathbf{v}_{h}, p\right), & & \forall \mathbf{v}_{h} \in \boldsymbol{V}_{h}, \\
m_{h}\left(\mathbf{w}_{\pi}, \mathbf{z}_{h}\right)-\left(\operatorname{div}_{h}, p_{\pi}\right) & =m\left(\mathbf{w}, \mathbf{z}_{h}\right)-\left(\operatorname{div} \mathbf{z}_{h}, p\right), & & \forall \mathbf{z}_{h} \in \mathbf{\Sigma}_{h}, \\
\left(\operatorname{div}_{\pi}, q_{h}\right) & =\left(\operatorname{div} \mathbf{w}, q_{h}\right), & & \forall q_{h} \in Q_{h} .
\end{aligned}
$$

It is easy to see that Theorems 3.8, 3.11 and 3.12 imply the well-posedness of the elliptic projection. If $\mathbf{u}, \mathbf{w}$ and $p$ are smooth enough, we also have the following estimates.

Theorem 4.2. Given $(\mathbf{u}, \mathbf{w}, p) \in \boldsymbol{V} \times \boldsymbol{\Sigma} \times Q$, let $\left(\mathbf{u}_{\pi}, \mathbf{w}_{\pi}, p_{\pi}\right) \in \boldsymbol{V}_{h} \times \boldsymbol{\Sigma}_{h} \times Q_{h}$ be the solution of (4.4)-(4.6). If $(\mathbf{u}, \mathbf{w}, p) \in\left[H^{k+1}(\Omega)\right]^{2} \times\left[H^{k}(\Omega)\right]^{2} \times H^{k}(\Omega)$ and $k \geq 2$, then the following estimates hold:

$$
\begin{aligned}
\left\|\mathbf{w}-\mathbf{w}_{\pi}\right\|_{0} & \leq C h^{k}\|\mathbf{w}\|_{k}, \\
\left\|p-p_{\pi}\right\|_{0} & \leq C h^{k}\left(\|\mathbf{w}\|_{k}+\|p\|_{k}\right), \\
\left\|\mathbf{u}-\mathbf{u}_{\pi}\right\|_{1} & \leq C h^{k}\left(\|\mathbf{u}\|_{k+1}+\|\lambda \operatorname{div} \mathbf{u}\|_{k}+\|\mathbf{w}\|_{k}+\|p\|_{k}\right) .
\end{aligned}
$$


Proof. We start with splitting errors as follows:

$$
\begin{aligned}
\mathbf{u}-\mathbf{u}_{\pi} & =\mathbf{u}-\Pi_{F} \mathbf{u}+\Pi_{F} \mathbf{u}-\mathbf{u}_{\pi}, \\
\mathbf{w}-\mathbf{w}_{\pi} & =\mathbf{w}-\Pi_{k}^{i} \mathbf{w}+\Pi_{k}^{i} \mathbf{w}-\mathbf{w}_{\pi}, \\
p-p_{\pi} & =p-\Pi_{k}^{0} p+\Pi_{k}^{0} p-p_{\pi} .
\end{aligned}
$$

Adding and subtracting proper term in (4.5), we have

$$
m_{h}\left(\mathbf{w}_{\pi}-\Pi_{k}^{i} \mathbf{w}, \mathbf{z}_{h}\right)-\left(\operatorname{div} \mathbf{z}_{h}, p_{\pi}-\Pi_{k}^{0} p\right)=m\left(\mathbf{w}-\Pi_{k}^{0} \mathbf{w}, \mathbf{z}_{h}\right)+m_{h}\left(\Pi_{k}^{0} \mathbf{w}-\Pi_{k}^{i} \mathbf{w}, \mathbf{z}_{h}\right)-\left(\operatorname{div} \mathbf{z}_{h}, p-\Pi_{k}^{0} p\right),
$$

where we have used the fact that $m\left(\Pi_{k}^{0} \mathbf{w}, \mathbf{z}_{h}\right)=m_{h}\left(\Pi_{k}^{0} \mathbf{w}, \mathbf{z}_{h}\right), \quad \forall \mathbf{z} \in \boldsymbol{\Sigma}_{h}$. Clearly, (4.6) and (3.14) means that

$$
\operatorname{div} \mathbf{w}_{\pi}=\Pi_{k}^{0} \operatorname{div} \mathbf{w}=\operatorname{div} \Pi_{k}^{i} \mathbf{w}
$$

from which we get

$$
\operatorname{div}\left(\mathbf{w}_{\pi}-\Pi_{k}^{i} \mathbf{w}\right)=0 .
$$

Then setting $\mathbf{z}_{h}=\mathbf{w}_{\pi}-\Pi_{k}^{i} \mathbf{w}$ in (4.10), using Theorem 3.12 and applying the Cauchy-Schwarz inequality, we obtain

$$
\left\|\mathbf{w}_{\pi}-\Pi_{k}^{i} \mathbf{w}\right\|_{0}^{2} \leq C\left(\left\|\mathbf{w}-\Pi_{k}^{0} \mathbf{w}\right\|_{0}\left\|\mathbf{w}_{\pi}-\Pi_{k}^{i} \mathbf{w}\right\|_{0}+\left\|\Pi_{k}^{0} \mathbf{w}-\Pi_{k}^{i} \mathbf{w}\right\|_{0}\left\|\mathbf{w}_{\pi}-\Pi_{k}^{i} \mathbf{w}\right\|_{0}\right) .
$$

Using the triangle inequality, (3.15) and (3.3) leads to (4.7). Applying Theorem 3.8, we can get $\overline{\mathbf{z}} \in \boldsymbol{\Sigma}_{h}$ such that

$$
\begin{aligned}
\operatorname{div} \overline{\mathbf{z}} & =-\left(p_{\pi}-\Pi_{k}^{0} p\right), \\
\|\overline{\mathbf{z}}\|_{\text {div }} & \leq C\left\|p_{\pi}-\Pi_{k}^{0} p\right\|_{0} .
\end{aligned}
$$

Testing such $\overline{\mathbf{z}}$ in (4.10) and using the Cauchy-Schwarz inequality, we have

$$
\left\|p_{\pi}-\Pi_{k}^{0} p\right\|_{0} \leq C\left(\left\|\mathbf{w}-\Pi_{k}^{0} \mathbf{w}\right\|_{0}+\left\|\Pi_{k}^{0} \mathbf{w}-\Pi_{k}^{i} \mathbf{w}\right\|_{0}+\left\|\Pi_{k}^{i} \mathbf{w}-\mathbf{w}_{\pi}\right\|_{0}\right) .
$$

Applying the triangle inequality, we deduce (4.8).

We now turn to the last estimate (4.9). Adding and subtracting $\Pi_{F} \mathbf{u}$ and $\Pi_{k}^{0} \mathbf{u}$ in (4.6), we have

$$
\begin{aligned}
\tilde{a}_{h}\left(\mathbf{u}_{\pi}-\Pi_{F} \mathbf{u}, \mathbf{v}_{h}\right) & +\lambda \sum_{E \in \mathcal{T}_{h}}\left(\Pi_{k-1}^{0, E} \operatorname{div}\left(\mathbf{u}-\Pi_{F} \mathbf{u}\right), \Pi_{k-1}^{0, E} \operatorname{div} \mathbf{v}_{h}\right)_{E}=\tilde{a}\left(\mathbf{u}_{\pi}-\Pi_{k}^{0} \mathbf{u}, \mathbf{v}_{h}\right) \\
& +\tilde{a}_{h}\left(\Pi_{k}^{0} \mathbf{u}_{-} \Pi_{F} \mathbf{u}, \mathbf{v}_{h}\right)+\alpha\left(\operatorname{div}_{h}, p-p_{\pi}\right)+\lambda\left(\operatorname{div} \mathbf{u}, \operatorname{div} \mathbf{v}_{h}\right) \\
& -\lambda \sum_{E \in \mathcal{T}_{h}}\left(\Pi_{k-1}^{0, E} \operatorname{div}\left(\Pi_{F} \mathbf{u}\right), \Pi_{k-1}^{0, E} \operatorname{div}_{h}\right)_{E} .
\end{aligned}
$$

We observe that (3.7) means $\Pi_{k-1}^{0, E} \operatorname{div}\left(\Pi_{F} \mathbf{u}\right)=\Pi_{k-1}^{0, E} \operatorname{div}(\mathbf{u})$. Then setting $\mathbf{v}_{h}=\mathbf{u}_{\pi}-\Pi_{F} \mathbf{u}$, we obtain

$$
\begin{aligned}
\tilde{a}_{h}\left(\mathbf{u}_{\pi}-\Pi_{F} \mathbf{u}, \mathbf{u}_{\pi}-\Pi_{F} \mathbf{u}\right) & +\lambda \sum_{E \in \mathcal{T}_{h}}\left(\Pi_{k-1}^{0, E} \operatorname{div}\left(\mathbf{u}-\Pi_{F} \mathbf{u}\right), \Pi_{k-1}^{0, E} \operatorname{div}\left(\mathbf{u}_{\pi}-\Pi_{F} \mathbf{u}\right)\right)_{E}=\tilde{a}\left(\mathbf{u}_{\pi}-\Pi_{k}^{0} \mathbf{u}, \mathbf{u}_{\pi}\right. \\
& \left.-\Pi_{F} \mathbf{u}\right)+\tilde{a}_{h}\left(\Pi_{k}^{0} \mathbf{u}-\Pi_{F} \mathbf{u}, \mathbf{u}_{\pi}-\Pi_{F} \mathbf{u}\right)+\alpha\left(\operatorname{div}\left(\mathbf{u}_{\pi}-\Pi_{F} \mathbf{u}\right), p-p_{\pi}\right) \\
& +\lambda \sum_{E \in \mathcal{T}_{h}}\left(\operatorname{div} \mathbf{u}-\Pi_{k-1}^{0, E} \operatorname{div}(\mathbf{u}), \operatorname{div}\left(\mathbf{u}_{\pi}-\Pi_{F} \mathbf{u}\right)\right)_{E}
\end{aligned}
$$


Using (3.25) and the Cauchy-Schwarz inequality, we get

$$
\begin{aligned}
\left\|\varepsilon\left(\mathbf{u}_{\pi}-\Pi_{F} \mathbf{u}\right)\right\|_{0}^{2} \leq & C\left(\left(\sum_{E \in \mathcal{T}_{h}}\left\|\nabla\left(\mathbf{u}_{\pi}-\Pi_{k}^{0} \mathbf{u}\right)\right\|_{0, E}^{2}\right)^{1 / 2}\left\|\nabla\left(\mathbf{u}_{\pi}-\Pi_{F} \mathbf{u}\right)\right\|_{0}\right. \\
& +\left(\sum_{E \in \mathcal{T}_{h}}\left\|\nabla\left(\Pi_{k}^{0} \mathbf{u}-\Pi_{F} \mathbf{u}\right)\right\|_{0, E}^{2}\right)^{1 / 2}\left\|\nabla\left(\mathbf{u}_{\pi}-\Pi_{F} \mathbf{u}\right)\right\|_{0}+\left\|\nabla\left(\mathbf{u}_{\pi}-\Pi_{F} \mathbf{u}\right)\right\|_{0}\left\|p-p_{\pi}\right\|_{0} \\
& \left.+\left(\lambda \sum_{E \in \mathcal{T}_{h}}\left\|\operatorname{div} \mathbf{u}-\Pi_{k-1}^{0, E} \operatorname{div}(\mathbf{u})\right\|_{0, E}^{2}\right)^{1 / 2}\left\|\nabla\left(\mathbf{u}_{\pi}-\Pi_{F} \mathbf{u}\right)\right\|_{0}\right) .
\end{aligned}
$$

Finally, using the Korn's inequality and the triangle inequality, we can derive (4.9).

With the above elliptic projection, we can define initial data as:

$$
\left(\mathbf{u}_{\pi}^{0}, \mathbf{w}_{\pi}^{0}, p_{\pi}^{0}\right)=\Pi_{e}\left(\mathbf{u}^{0}, \mathbf{w}^{0}, p^{0}\right) .
$$

\subsection{A priori error estimates for $c_{0}=0$}

In this section, we will derive a priori error estimates for the discrete problem (4.1)-(4.3) with $c_{0}=0$. According to Phillips and Wheeler [52], the poroelastic locking typically occurs when the constrained specific storage term is null $\left(c_{0}=0\right)$, the permeability of the porous medium is very low, and a small time step is used. Therefore we are more interested in the error estimate in the case $c_{0}=0$. For $c_{0}>0$, we can apply the argument in [61] to get desired a priori error estimates. To prove optimal error estimates, we need the following regularity assumptions:

$$
\begin{aligned}
\mathbf{u} & \in L^{\infty}\left(\left[0, T_{0}\right] ; H^{k+1}(\Omega)\right), & & \mathbf{f}, \lambda \operatorname{div} \mathbf{u}, \mathbf{w}, p \in L^{\infty}\left(\left[0, T_{0}\right] ; H^{k}(\Omega)\right), \\
\mathbf{u}_{t} & \in L^{2}\left(\left[0, T_{0}\right] ; H^{k+1}(\Omega)\right), & & \mathbf{f}_{t}, \lambda \operatorname{div} \mathbf{u}_{t}, \mathbf{w}_{t}, p_{t} \in L^{2}\left(\left[0, T_{0}\right] ; H^{k}(\Omega)\right), \\
\mathbf{u}_{t t} & \in L^{2}\left(\left[0, T_{0}\right] ; H^{1}(\Omega)\right), & & \mathbf{f}_{t t} \in L^{2}\left(\left[0, T_{0}\right] ; L^{2}(\Omega)\right) .
\end{aligned}
$$

The main results are collected in the following theorem.

Theorem 4.3. Let $(\mathbf{u}, \mathbf{w}, p)$ be the solution of $(2.7)-(2.9)$ and $\left(\mathbf{u}_{h}^{n}, \mathbf{w}_{h}^{n}, p_{h}^{n}\right)$ the solution of fully discrete problem (4.1)-(4.3). Assume that $k \geq 2$ and (4.12) hold. Then we have the following error estimates:

$$
\max _{1 \leq n \leq N}\left(\left\|\mathbf{u}^{n}-\mathbf{u}_{h}^{n}\right\|_{1}\right)^{2}+\Delta t \sum_{n=0}^{N}\left(\left\|\mathbf{w}^{n}-\mathbf{w}_{h}^{n}\right\|_{0}^{2}+\left\|p^{n}-p_{h}^{n}\right\|_{0}^{2}\right) \leq C\left(h^{2 k}+\Delta t^{2}\right),
$$

where $C$ is a positive constant independent of $h$ and $\lambda$.

Proof. We start the proof with recalling the following Taylor expansion:

$$
\frac{\mathbf{u}^{n}-\mathbf{u}^{n-1}}{\Delta t}=\mathbf{u}_{t}^{n}+\frac{1}{\Delta t} \int_{t^{n-1}}^{t^{n}}\left(s-t^{n-1}\right) \mathbf{u}_{t t}(s) \mathrm{d} s .
$$

Then, at time $t=t^{n}$, using the above equation in (2.7)-(2.9), we have

$$
\begin{aligned}
a\left(\mathbf{u}^{n}, \mathbf{v}_{h}\right)-\alpha\left(p^{n}, \operatorname{div}_{h}\right) & =\left(\mathbf{f}^{n}, \mathbf{v}_{h}\right), \\
m\left(\mathbf{w}^{n}, \mathbf{z}_{h}\right)-\left(p^{n}, \operatorname{div} \mathbf{z}_{h}\right) & =0, \\
\alpha\left(\operatorname{div}\left(\frac{\mathbf{u}^{n}-\mathbf{u}^{n-1}}{\Delta t}\right), q_{h}\right)+\left(\operatorname{div}^{n}, q_{h}\right) & =\left(g^{n}, q_{h}\right)+\frac{\alpha}{\Delta t}\left(\int_{t^{n-1}}^{t^{n}}\left(s-t^{n-1}\right) \operatorname{div} \mathbf{u}_{t t}(s) \mathrm{d} s, q_{h}\right),
\end{aligned}
$$


for all $\mathbf{v}_{h} \in \boldsymbol{V}_{h}, \mathbf{z}_{h} \in \boldsymbol{\Sigma}_{h}, q_{h} \in Q_{h}$.

Applying elliptic projection (4.4)-(4.6), we obtain

$$
\begin{aligned}
a_{h}\left(\Pi_{e} \mathbf{u}^{n}, \mathbf{v}_{h}\right)-\alpha\left(\Pi_{e} p^{n}, \operatorname{div} \mathbf{v}_{h}\right) & =\left(\mathbf{f}^{n}, \mathbf{v}_{h}\right), \\
m_{h}\left(\Pi_{e} \mathbf{w}^{n}, \mathbf{z}_{h}\right)-\left(\Pi_{e} p^{n}, \operatorname{div} \mathbf{z}_{h}\right) & =0, \\
\alpha\left(\operatorname{div}\left(\frac{\mathbf{u}^{n}-\mathbf{u}^{n-1}}{\Delta t}\right), q_{h}\right)+\left(\operatorname{div} \Pi_{e} \mathbf{w}^{n}, q_{h}\right) & =\left(g^{n}, q_{h}\right)+\frac{\alpha}{\Delta t}\left(\int_{t^{n-1}}^{t^{n}}\left(s-t^{n-1}\right) \operatorname{div} \mathbf{u}_{t t}(s) \mathrm{d} s, q_{h}\right),
\end{aligned}
$$

for all $\mathbf{v}_{h} \in \boldsymbol{V}_{h}, \mathbf{z}_{h} \in \boldsymbol{\Sigma}_{h}, q_{h} \in Q_{h}$. Next we split errors as follows:

$$
\begin{aligned}
\mathbf{u}^{n}-\mathbf{u}_{h}^{n} & =\mathbf{u}^{n}-\Pi_{e} \mathbf{u}^{n}+\Pi_{e} \mathbf{u}^{n}-\mathbf{u}_{h}^{n}:=\boldsymbol{\eta}_{u}^{n}+\boldsymbol{\xi}_{u}^{n}, \\
\mathbf{w}^{n}-\mathbf{w}_{h}^{n} & =\mathbf{w}^{n}-\Pi_{e} \mathbf{w}^{n}+\Pi_{e} \mathbf{w}^{n}-\mathbf{w}_{h}^{n}:=\boldsymbol{\eta}_{w}^{n}+\boldsymbol{\xi}_{w}^{n}, \\
p^{n}-p_{h}^{n} & =p^{n}-\Pi_{e} p^{n}+\Pi_{e} p^{n}-p_{h}^{n}:=\eta_{p}^{n}+\xi_{p}^{n} .
\end{aligned}
$$

By taking the differences of (4.14)-(4.16) and (4.1)-(4.3), we have

$$
\begin{aligned}
a_{h}\left(\boldsymbol{\xi}_{u}^{n}, \mathbf{v}_{h}\right)-\alpha\left(\xi_{p}^{n}, \operatorname{div}_{h}\right) & =\left(R_{f}^{n}, \mathbf{v}_{h}\right), \mathbf{v}_{h} \in \boldsymbol{V}_{h}, \\
m_{h}\left(\boldsymbol{\xi}_{w}^{n}, \mathbf{z}_{h}\right)-\left(\xi_{p}^{n}, \operatorname{div}_{h}\right) & =0, \mathbf{z}_{h} \in \mathbf{\Sigma}_{h}, \\
\alpha\left(\operatorname{div}\left(\frac{\boldsymbol{\xi}_{u}^{n}-\boldsymbol{\xi}_{u}^{n-1}}{\Delta t}\right), q_{h}\right)+\left(\operatorname{div} \xi_{w}^{n}, q_{h}\right) & =-\alpha\left(\operatorname{div}\left(\frac{\boldsymbol{\eta}_{u}^{n}-\boldsymbol{\eta}_{u}^{n-1}}{\Delta t}\right), q_{h}\right)+\frac{\alpha}{\Delta t}\left(R_{u}^{n}, q_{h}\right), q_{h} \in Q_{h},
\end{aligned}
$$

where $R_{f}^{n}=f^{n}-\Pi_{k}^{0} f^{n}, R_{u}^{n}=\int_{t^{n-1}}^{t^{n}}\left(s-t^{n-1}\right) \operatorname{div} \mathbf{u}_{t t}(s) \mathrm{d} s$. Taking $\mathbf{v}_{h}=\frac{\boldsymbol{\xi}_{u}^{n}-\boldsymbol{\xi}_{u}^{n-1}}{\Delta t}, \mathbf{z}_{h}=\boldsymbol{\xi}_{w}^{n}$ and $q_{h}=\xi_{p}^{n}$ in the above equations leads to

$$
a_{h}\left(\boldsymbol{\xi}_{u}^{n}, \boldsymbol{\xi}_{u}^{n}-\boldsymbol{\xi}_{u}^{n-1}\right)+\Delta t m_{h}\left(\xi_{w}^{n}, \xi_{w}^{n}\right)=-\alpha\left(\operatorname{div}\left(\boldsymbol{\eta}_{u}^{n}-\boldsymbol{\eta}_{u}^{n-1}\right), \xi_{p}^{n}\right)+\alpha\left(R_{u}^{n}, \xi_{p}^{n}\right)+\left(R_{f}^{n}, \boldsymbol{\xi}_{u}^{n}-\boldsymbol{\xi}_{u}^{n-1}\right) .
$$

Let $\left\|\boldsymbol{\xi}_{u}^{n}\right\|_{a_{h}}^{2}:=a_{h}\left(\boldsymbol{\xi}_{u}^{n}, \boldsymbol{\xi}_{u}^{n}\right)$ and $\left\|\xi_{w}^{n}\right\|_{m_{h}}^{2}:=m_{h}\left(\xi_{w}^{n}, \xi_{w}^{n}\right)$. Observing that

$$
a_{h}\left(\boldsymbol{\xi}_{u}^{n}, \boldsymbol{\xi}_{u}^{n-1}\right) \leq\left\|\boldsymbol{\xi}_{u}^{n}\right\|_{a_{h}}\left\|\boldsymbol{\xi}_{u}^{n-1}\right\|_{a_{h}} \leq \frac{\left\|\boldsymbol{\xi}_{u}^{n}\right\|_{a_{h}}^{2}+\left\|\boldsymbol{\xi}_{u}^{n-1}\right\|_{a_{h}}^{2}}{2}
$$

we can rewrite (4.20) as

$$
\frac{1}{2}\left(\left\|\boldsymbol{\xi}_{u}^{n}\right\|_{a_{h}}^{2}-\left\|\boldsymbol{\xi}_{u}^{n-1}\right\|_{a_{h}}^{2}\right)+\Delta t\left\|\xi_{w}^{n}\right\|_{m_{h}}^{2} \leq-\alpha\left(\operatorname{div}\left(\boldsymbol{\eta}_{u}^{n}-\boldsymbol{\eta}_{u}^{n-1}\right), \xi_{p}^{n}\right)+\alpha\left(R_{u}^{n}, \xi_{p}^{n}\right)+\left(R_{f}^{n}, \boldsymbol{\xi}_{u}^{n}-\boldsymbol{\xi}_{u}^{n-1}\right) .
$$

Summing it from 1 to $M(1 \leq M \leq N)$ yields

$$
\frac{1}{2}\left\|\boldsymbol{\xi}_{u}^{M}\right\|_{a_{h}}^{2}+\sum_{n=1}^{M} \Delta t\left\|\xi_{w}^{n}\right\|_{m_{h}}^{2} \leq C\left(R_{1}+R_{2}+R_{3}\right),
$$

where

$$
\begin{aligned}
& R_{1}=-\alpha \sum_{n=1}^{M}\left(\operatorname{div}\left(\boldsymbol{\eta}_{u}^{n}-\boldsymbol{\eta}_{u}^{n-1}\right), \xi_{p}^{n}\right), \\
& R_{2}=\alpha \sum_{n=1}^{M}\left(R_{u}^{n}, \xi_{p}^{n}\right), \\
& R_{3}=\sum_{n=1}^{M}\left(R_{f}^{n}, \boldsymbol{\xi}_{u}^{n}-\boldsymbol{\xi}_{u}^{n-1}\right) .
\end{aligned}
$$


Now our main task is to estimate $R_{1}, R_{2}$ and $R_{3}$. To this end, we use (4.13) to rewrite $R_{1}$ as

$$
R_{1}=\sum_{n=1}^{M}\left(\left(\Delta t \operatorname{div} \boldsymbol{\eta}_{u_{t}}^{n}, \xi_{p}^{n}\right)+\left(\int_{t^{n-1}}^{t^{n}}\left(s-t^{n-1}\right) \operatorname{div} \boldsymbol{\eta}_{u_{t t}}(s) \mathrm{d} s, \xi_{p}^{n}\right)\right) .
$$

Using Young's inequality yields

$$
\begin{aligned}
\left|R_{1}\right| & \leq \sum_{n=1}^{M}\left(\frac{1}{2 \epsilon_{1}}\left\|\operatorname{div} \boldsymbol{\eta}_{u_{t}}^{n}\right\|_{0}^{2} \Delta t+\epsilon_{1}\left\|\xi_{p}^{n}\right\|_{0}^{2} \Delta t+\frac{1}{2 \epsilon_{1} \Delta t}\left\|\int_{t^{n-1}}^{t^{n}}\left(s-t^{n-1}\right) \operatorname{div} \boldsymbol{\eta}_{u_{t t}}(s)\right\|_{0}\right) \\
& \leq C\left(\sum_{n=1}^{M}\left\|\boldsymbol{\eta}_{u_{t}}^{n}\right\|_{1}^{2} \Delta t+\Delta t^{2} \sum_{n=1}^{M} \int_{t^{n-1}}^{t^{n}}\left\|\boldsymbol{\eta}_{u_{t t}}(s)\right\|_{1}^{2} \mathrm{~d} s+\epsilon_{1} \sum_{n=1}^{M}\left\|\boldsymbol{\xi}_{w}^{n}\right\|_{0}^{2} \Delta t\right),
\end{aligned}
$$

where we have used Theorem 3.8 and (4.18) to get

$$
\left\|\xi_{p}^{n}\right\|_{0} \leq C \sup _{\substack{\mathbf{z}_{h} \in \Sigma_{h}, \mathbf{z}_{h} \neq 0}} \frac{\left(\operatorname{div} \mathbf{z}_{h}, \xi_{p}^{n}\right)}{\left\|\mathbf{z}_{h}\right\|_{\text {div }}}=C \sup _{\substack{\mathbf{z}_{h} \in \Sigma_{h}, \mathbf{z}_{h} \neq 0}} \frac{m_{h}\left(\boldsymbol{\xi}_{w}^{n}, \mathbf{z}_{h}\right)}{\left\|\mathbf{z}_{h}\right\|_{\text {div }}} \leq C\left\|\boldsymbol{\xi}_{w}^{n}\right\|_{0} .
$$

Using Young's inequality again, we obtain

$$
\left|R_{2}\right| \leq C \sum_{n=1}^{M} \frac{1}{\Delta t}\left\|R_{u}^{n}\right\|_{0}^{2}+\epsilon_{1} \sum_{n=1}^{M}\left\|\boldsymbol{\xi}_{w}^{n}\right\|_{0}^{2} \Delta t
$$

Since

$$
\left|R_{u}^{n}\right| \leq\left(\int_{t^{n-1}}^{t^{n}}\left|s-t^{n-1}\right|^{2} \mathrm{~d} s\right)^{1 / 2}\left(\int_{t^{n-1}}^{t^{n}}\left|\operatorname{div} \mathbf{u}_{t t}(s)\right|^{2} \mathrm{~d} s\right)^{1 / 2} \leq \frac{\Delta t^{3 / 2}}{\sqrt{3}}\left(\int_{t^{n-1}}^{t^{n}}\left|\operatorname{div} \mathbf{u}_{t t}(s)\right|^{2} \mathrm{~d} s\right)^{1 / 2}
$$

we have

$$
\left|R_{2}\right| \leq C \Delta t^{2} \sum_{n=1}^{M} \int_{t^{n-1}}^{t^{n}}\left\|\mathbf{u}_{t t}(s)\right\|_{1}^{2} \mathrm{~d} s+\epsilon_{1} \sum_{n=1}^{M}\left\|\boldsymbol{\xi}_{w}^{n}\right\|_{0}^{2} \Delta t .
$$

Note that the following equation holds for any bounded sequences $\left\{f^{i}\right\}_{i=0}^{M}$ and $\left\{g^{i}\right\}_{i=0}^{M}$

$$
\sum_{n=1}^{M} f^{n}\left(g^{n}-g^{n-1}\right)=f^{M} g^{M}-f^{0} g^{0}-\sum_{n=1}^{M}\left(f^{n}-f^{n-1}\right) g^{n-1} .
$$

By using the above equation, $R_{3}$ can be rewritten as

$$
R_{3}=\left(R_{f}^{M}, \boldsymbol{\xi}_{u}^{M}\right)-\sum_{n=1}^{M}\left(R_{f}^{n}-R_{f}^{n-1}, \boldsymbol{\xi}_{u}^{n-1}\right)
$$

Applying (4.13) to $R_{f}^{n}-R_{f}^{n-1}$, we can estimate $R_{3}$ as follows

$$
\left|R_{3}\right| \leq C\left\|R_{f}^{M}\right\|_{0}^{2}+\frac{\epsilon_{1}}{2}\left\|\boldsymbol{\xi}_{u}^{M}\right\|_{1}^{2}+C \sum_{n=1}^{M}\left\|R_{f}^{n}\right\|_{0}^{2} \Delta t+\epsilon_{1} \sum_{n=1}^{M}\left\|\boldsymbol{\xi}_{u}^{n-1}\right\|_{1}^{2} \Delta t+C \Delta t^{2} \sum_{n=1}^{M} \int_{t^{n-1}}^{t^{n}}\left\|R_{f_{t t}}(s)\right\|_{0}^{2} \mathrm{~d} s .
$$


Combing (4.21), (4.23), (4.25) and (4.26), choosing $\epsilon_{1}$ properly, and using (3.31) and Theorem 3.11, we obtain

$$
\begin{aligned}
\mu\left\|\boldsymbol{\xi}_{u}^{M}\right\|_{1}^{2}+\sum_{n=0}^{M}\left\|\boldsymbol{\xi}_{w}^{n}\right\|_{0}^{2} \Delta t \leq & C\left(\left\|R_{f}^{M}\right\|_{0}^{2}+\sum_{n=1}^{M}\left\|R_{f_{t}}^{n}\right\|_{0}^{2} \Delta t\right. \\
& +\sum_{n=1}^{M} \Delta t^{2} \int_{t^{n-1}}^{t_{n}}\left\|R_{f_{t t}}(s)\right\|_{0}^{2} \mathrm{~d} s+\sum_{n=1}^{M}\left\|\boldsymbol{\eta}_{u_{t}}\right\|_{1}^{2} \Delta t+\Delta t^{2} \sum_{n=1}^{M} \int_{t^{n-1}}^{t^{n}}\left\|\boldsymbol{\eta}_{u_{t t}}(s)\right\|_{1}^{2} \mathrm{~d} s \\
& \left.+\Delta t^{2} \sum_{n=1}^{M} \int_{t^{n-1}}^{t^{n}}\left\|\mathbf{u}_{t t}(s)\right\|_{1}^{2} \mathrm{~d} s\right)+\sum_{n=0}^{N-1}\left\|\boldsymbol{\xi}_{u}^{n}\right\|_{1}^{2} \Delta t .
\end{aligned}
$$

Using discrete Gronwall inequality yields

$$
\begin{aligned}
\mu\left\|\boldsymbol{\xi}_{u}^{M}\right\|_{1}^{2}+\sum_{n=0}^{M}\left\|\boldsymbol{\xi}_{w}^{n}\right\|_{0}^{2} \Delta t \leq & C \exp \left(T_{0}\right)\left(\left\|R_{f}^{N}\right\|_{0}^{2}+\sum_{n=1}^{N}\left\|R_{f_{t}}^{n}\right\|_{0}^{2} \Delta t\right. \\
& \left.+\sum_{n=1}^{N}\left\|\boldsymbol{\eta}_{u_{t}}^{n}\right\|_{1}^{2} \Delta t+\Delta t^{2} \int_{0}^{T_{0}}\left\|\mathbf{f}_{t t}(s)\right\|_{0}^{2} \mathrm{~d} s+\Delta t^{2} \int_{0}^{T_{0}}\left\|\mathbf{u}_{t t}(s)\right\|_{1}^{2} \mathrm{~d} s\right) .
\end{aligned}
$$

It follows from (3.3) that

$$
\left\|R_{f}^{N}\right\|_{0} \leq C h^{k}\|\mathbf{f}\|_{L^{\infty}\left(\left[0, T_{0}\right] ; H^{k}\right)} .
$$

Let $s \in\left[t_{n-1}, t_{n}\right]$, then we can estimate $\left\|R_{f_{t}}^{n}\right\|_{0}$ as follows

$$
\left\|R_{f_{t}}^{n}\right\|_{0} \leq\left\|\left(I-\Pi_{k}^{0}\right)\left(\mathbf{f}_{t}^{n}-\mathbf{f}_{t}(s)\right)\right\|_{0}+\left\|\left(I-\Pi_{k}^{0}\right)\left(\mathbf{f}_{t}(s)\right)\right\|_{0} \leq\left\|\mathbf{f}_{t}^{n}-\mathbf{f}_{t}(s)\right\|_{0}+\left\|\left(I-\Pi_{k}^{0}\right)\left(\mathbf{f}_{t}(s)\right)\right\|_{0} .
$$

Noting

$$
\mathbf{f}_{t}^{n}-\mathbf{f}_{t}(s)=\int_{s}^{t_{n}} \mathbf{f}_{t t}(r) \mathrm{d} r
$$

we have

$$
\left\|\mathbf{f}_{t}^{n}-\mathbf{f}_{t}(s)\right\|_{0}^{2} \leq \Delta t \int_{t_{n-1}}^{t_{n}}\left\|\mathbf{f}_{t t}(r)\right\|_{0}^{2} \mathrm{~d} r
$$

From (3.3) we obtain

$$
\left\|\left(I-\Pi_{k}^{0}\right)\left(\mathbf{f}_{t}(s)\right)\right\|_{0} \leq C h^{k}\left\|\mathbf{f}_{t}(s)\right\|_{k} .
$$

Combing (4.27)-(4.29), we derive

$$
\sum_{n=1}^{N}\left\|R_{f_{t}}^{n}\right\|_{0}^{2} \Delta t \leq C \Delta t^{2} \int_{0}^{T_{0}}\left\|\mathbf{f}_{t t}(r)\right\|_{0}^{2} \mathrm{~d} r+C h^{2 k} \int_{0}^{T_{0}}\left\|\mathbf{f}_{t}(s)\right\|_{k}^{2} \mathrm{~d} s .
$$

By the same token, we have

$$
\begin{aligned}
\sum_{n=1}^{N}\left\|\boldsymbol{\eta}_{u_{t}}\right\|_{1}^{2} \Delta t \leq & C \Delta t^{2} \int_{0}^{T_{0}}\left\|\mathbf{u}_{t t}(s)\right\|_{1}^{2} \mathrm{~d} s+C h^{2 k}\left(\int_{0}^{T_{0}}\left\|\mathbf{u}_{t}(s)\right\|_{k+1}^{2} \mathrm{~d} s\right. \\
& \left.+\int_{0}^{T_{0}}\left\|\lambda \operatorname{div} \mathbf{u}_{t}(s)\right\|_{k}^{2} \mathrm{~d} s+\int_{0}^{T_{0}}\left\|\mathbf{w}_{t}(s)\right\|_{k}^{2} \mathrm{~d} s+\int_{0}^{T_{0}}\left\|p_{t}(s)\right\|_{k}^{2} \mathrm{~d} s\right) .
\end{aligned}
$$


As a consequence, we arrive at

$$
\begin{aligned}
\mu\left\|\boldsymbol{\xi}_{u}^{M}\right\|_{1}^{2}+\sum_{n=0}^{M}\left\|\boldsymbol{\xi}_{w}^{n}\right\|_{0}^{2} \Delta t \leq & C \exp \left(T_{0}\right)\left(\Delta t^{2}\left(\int_{0}^{T_{0}}\left\|\mathbf{f}_{t t}(s)\right\|_{0}^{2} \mathrm{~d} s+\int_{0}^{T_{0}}\left\|\mathbf{u}_{t t}(s)\right\|_{1}^{2} \mathrm{~d} s\right)\right. \\
& +h^{2 k}\left(h^{2 k}\|\mathbf{f}\|_{L^{\infty}\left(\left[0, T_{0}\right] ; H^{k}\right)}+\int_{0}^{T_{0}}\left\|\mathbf{f}_{t}(s)\right\|_{k}^{2} \mathrm{~d} s+\int_{0}^{T_{0}}\left\|\mathbf{u}_{t}(s)\right\|_{k+1}^{2} \mathrm{~d} s\right. \\
& \left.\left.+\int_{0}^{T_{0}}\left\|\lambda \operatorname{div} \mathbf{u}_{t}(s)\right\|_{k}^{2} \mathrm{~d} s+\int_{0}^{T_{0}}\left\|\mathbf{w}_{t}(s)\right\|_{k}^{2} \mathrm{~d} s+\int_{0}^{T_{0}}\left\|p_{t}(s)\right\|_{k}^{2} \mathrm{~d} s\right)\right)
\end{aligned}
$$

Applying (4.24) yields

$$
\begin{aligned}
\sum_{n=0}^{N}\left\|\xi_{p}^{n}\right\|_{0}^{2} \Delta t \leq & C \exp \left(T_{0}\right)\left(\Delta t^{2}\left(\int_{0}^{T_{0}}\left\|\mathbf{f}_{t t}(s)\right\|_{0}^{2} \mathrm{~d} s+\int_{0}^{T_{0}}\left\|\mathbf{u}_{t t}(s)\right\|_{1}^{2} \mathrm{~d} s\right)\right. \\
& +h^{2 k}\left(h^{2 k}\|\mathbf{f}\|_{L^{\infty}\left(0, T_{0}, H^{k}\right)}+\int_{0}^{T_{0}}\left\|\mathbf{f}_{t}(s)\right\|_{k}^{2} \mathrm{~d} s+\int_{0}^{T_{0}}\left\|\mathbf{u}_{t}(s)\right\|_{k+1}^{2} \mathrm{~d} s\right. \\
& \left.\left.+\int_{0}^{T_{0}}\left\|\lambda \operatorname{div}_{t}(s)\right\|_{k}^{2} \mathrm{~d} s+\int_{0}^{T_{0}}\left\|\mathbf{w}_{t}(s)\right\|_{k}^{2} \mathrm{~d} s+\int_{0}^{T_{0}}\left\|p_{t}(s)\right\|_{k}^{2} \mathrm{~d} s\right)\right) .
\end{aligned}
$$

It follows from (4.9) that

$$
\left\|\boldsymbol{\eta}_{u}^{N}\right\|_{0} \leq C h^{k}\left(\|\mathbf{u}\|_{L^{\infty}\left(\left[0, T_{0}\right] ; H^{k+1}\right)}+\|\lambda \operatorname{div} \mathbf{u}\|_{L^{\infty}\left(\left[0, T_{0}\right] ; H^{k}\right)}+\|\mathbf{w}\|_{L^{\infty}\left(\left[0, T_{0}\right] ; H^{k}\right)}+\|p\|_{L^{\infty}\left(\left[0, T_{0}\right] ; H^{k}\right)}\right) .
$$

By similar procedure as in (4.27)-(4.29), we can get

$$
\begin{aligned}
\sum_{n=0}^{N}\left\|\boldsymbol{\eta}_{w}^{n}\right\|_{0}^{2} \Delta t \leq & C \Delta t^{2} \int_{0}^{T_{0}}\left\|\mathbf{w}_{t}(s)\right\|_{0}^{2} \mathrm{~d} s+C h^{2 k} \int_{0}^{T_{0}}\|\mathbf{w}(s)\|_{k}^{2} \mathrm{~d} s \\
\sum_{n=0}^{N}\left\|\eta_{p}^{n}\right\|_{0}^{2} \Delta t \leq & C \Delta t^{2}\left(\int_{0}^{T_{0}}\left\|p_{t}(s)\right\|_{0}^{2} \mathrm{~d} s+\int_{0}^{T_{0}}\left\|\mathbf{w}_{t}(s)\right\|_{0}^{2} \mathrm{~d} s\right) \\
& +C h^{2 k}\left(\int_{0}^{T_{0}}\|p(s)\|_{k}^{2} \mathrm{~d} s+\int_{0}^{T_{0}}\|\mathbf{w}(s)\|_{k}^{2} \mathrm{~d} s\right) .
\end{aligned}
$$

Finally, combing (4.30)-(4.34), and using the triangle inequality, we complete the proof.

Remark 4.4. Conditions $k \geq 2$ and (4.12) are the key to establish robust error estimates when $\lambda \rightarrow \infty$. If $k=1$, then Lemma 3.6 may no longer hold. As a consequence, we can only derive error bounds $C_{\lambda}\left(h^{2}+\Delta t^{2}\right)$, where $C_{\lambda}$ is dependent on $\lambda$.

Remark 4.5. Even though, Lemma 3.6 for $k=1$ does not hold on arbitrary mesh, we observe in numerical tests that for $k=1$, fully discrete scheme (4.1)-(4.3) seems to be locking-free on most types of meshes except triangular meshes. This implies that Lemma 3.6 may hold on some meshes, which reminds us of the work of [7]. Reference [7] proved that the mimetic generalization of the $P_{1}-P_{0}$ finite element is stable on a large range of polygonal meshes. Such mimetic generalization has the same degrees of freedom as that of the VEM when $k=1$. Therefore it is possible to translate the result to a VEM setting. However, we will not discuss this problem at present. We are more interested in developing a low-order locking-free VEM for Biot's model on general mesh. 
Remark 4.6. According to Theorem 2.1 and Remark 2.2, $\sup _{0 \leq t \leq T_{0}}\|\lambda \operatorname{div} \mathbf{u}(t)\|_{1}$ and $\sup _{0 \leq t \leq T_{0}}\left\|\lambda \operatorname{div} \mathbf{u}_{t}(t)\right\|_{1}$ can be bounded by $\mathbf{f}, g$ and initial data. Therefore, for $k \geq 2$, with mild modification of the proof, at least, we have

$$
\max _{1 \leq n \leq N}\left(\left\|\mathbf{u}^{n}-\mathbf{u}_{h}^{n}\right\|_{1}\right)^{2}+\Delta t \sum_{n=0}^{N}\left(\left\|\mathbf{w}^{n}-\mathbf{w}_{h}^{n}\right\|_{0}^{2}+\left\|p^{n}-p_{h}^{n}\right\|_{0}^{2}\right) \leq C\left(h^{2}+\Delta t^{2}\right),
$$

where $C$ is a positive constant independent of $h$ and $\lambda$.

The above discussion motivates us to establish a low-order locking-free VEM for Biot's model. We will introduce a possible scheme in the next section.

\subsection{A low order scheme}

From previous section, we observe that a key point to obtain robust estimates when $\lambda \rightarrow \infty$ is to construct a Fortin operator like Lemma 3.6. In this section, we concentrate on introducing a low order virtual element subspace that allows such operator. Our new virtual element is inspired by the classical Bernardi-Raugel elements introduced in [20]. The main idea is to introduce extra degrees of freedom related to the normal component of $\mathbf{v}$ on each edge. Most of the materials in this section are stated without proof. Related proofs can be founded in our recent work [56]. We just apply the results to Biot's consolidation model in poroelasticity.

We start with introducing some notations and recall virtual element subspaces defined in (3.4). For $E \in \mathcal{T}_{h}$, let $N_{e}^{E}$ be the number of the edges of $E$. We denote by $\mathbf{n}_{i}$ the unit outward normal vector to edge $e_{i}, 1 \leq i \leq N_{e}^{E}$. Next, for $1 \leq i, j \leq N_{e}^{E}$, let $\psi_{i} \in V_{2}^{E}$ satisfies

$$
\left|e_{j}\right|^{-1} \int_{e_{j}} \psi_{i}=\delta_{i j}, \quad \widetilde{\mathbf{D}}_{\mathbf{V}} \mathbf{1}\left(\psi_{i}\right)=0, \quad \widetilde{\mathbf{D}}_{\mathbf{V}} \mathbf{3}\left(\psi_{i}\right)=0
$$

where $\delta_{i j}$ is Kronecker delta. Then set

$$
\boldsymbol{\theta}_{i}:=\mathbf{n}_{i} \psi_{i}
$$

and let

$$
\widetilde{\boldsymbol{V}}_{2}^{E}:=\operatorname{span}\left\{\boldsymbol{\theta}_{i} 1 \leq i \leq N_{e}^{E}\right\} .
$$

We are now in a position to define the Bernardi-Raugel-like virtual element space: for $E \in \mathcal{T}_{h}$

$$
\boldsymbol{V}_{\mathrm{BR}}^{E}:=\left[V_{1}^{E}\right]^{2} \oplus \widetilde{\boldsymbol{V}}_{2}^{E}
$$

Clearly, $\operatorname{dim}\left(\boldsymbol{V}_{\mathrm{BR}}^{E}\right)=3 N_{e}^{E}$. The degrees of freedom we take for $\boldsymbol{V}_{\mathrm{BR}}^{E}$ are: for each $\mathbf{v} \in \boldsymbol{V}_{\mathrm{BR}}^{E}$

- $\mathbf{D}_{\mathbf{V}} \mathbf{1}$ : the values of two components of $\mathbf{v}$ at each vertex of $E$,

- $\mathbf{D}_{\mathbf{V}} \mathbf{2}$ : the values of the lowest moment of the normal component of $\mathbf{v}$ on each edge of $E$,

$$
\int_{e_{i}} \mathbf{v} \cdot \mathbf{n}_{i} \mathrm{~d} s \quad 1 \leq i \leq N_{e}^{E}
$$

The next lemma checks the unisolvence of these degrees of freedom [56].

Lemma 4.7. A vector-valued function $\mathbf{v} \in \boldsymbol{V}_{\mathrm{BR}}^{E}$ is uniquely determined by $\mathbf{D}_{\mathbf{V}} \mathbf{1}$ and $\mathbf{D}_{\mathbf{V}} \mathbf{2}$.

Remark 4.8. We emphasize that the idea of adding $\mathbf{D}_{\mathbf{V}} \mathbf{2}$ was introduced in [3]. Here we use different lifting inside in the definition of the space. Differently from [3], the proposed virtual element space are built upon [2], so the proposed method allows the explicit computation of local $L^{2}$ projection $\Pi_{k}^{0, E} \mathbf{v}$ from the knowledge of the degrees of freedom of $\mathbf{v}$. 
We now define the global virtual element spaces as

$$
\begin{aligned}
\boldsymbol{V}_{\mathrm{BR}}: & =\left\{\mathbf{v} \in\left[H^{1}(\Omega)\right]^{2}:\left.\mathbf{v}\right|_{E} \in \boldsymbol{V}_{\mathrm{BR}}^{E} \quad \forall E \in \mathcal{T}_{h}\right\}, \\
\widetilde{\boldsymbol{V}}_{2}: & =\left\{\mathbf{v} \in\left[H^{1}(\Omega)\right]^{2}:\left.\mathbf{v}\right|_{E} \in \widetilde{\boldsymbol{V}}_{2}^{E} \quad \forall E \in \mathcal{T}_{h}\right\} .
\end{aligned}
$$

It is easy to see that

$$
\boldsymbol{V}_{\mathrm{BR}}=\left[V_{1}\right]^{2} \oplus \tilde{\boldsymbol{V}}_{2}
$$

We now set

$$
\boldsymbol{V}_{h}:=\boldsymbol{V}_{\mathrm{BR}} \cap \boldsymbol{V}, \quad \boldsymbol{\Sigma}_{h}:=\Sigma_{h}^{0} \cap \boldsymbol{\Sigma}, \quad Q_{h}:=\mathbb{P}_{0}\left(\mathcal{T}_{h}\right) .
$$

We established the following theorem in [56].

Theorem 4.9. There exists an interpolation operator $I_{u}:\left[H_{0}^{1}(\Omega)\right]^{2} \rightarrow \boldsymbol{V}_{h}$ satisfying

$$
\left(\operatorname{div}\left(\mathbf{v}-I_{u} \mathbf{v}\right), q_{h}\right)=0 \quad \forall \mathbf{v} \in\left[H_{0}^{1}(\Omega)\right]^{2} \quad \forall q_{h} \in \mathbb{P}_{0}\left(\mathcal{T}_{h}\right)
$$

and

$$
\left|\mathbf{v}-I_{u} \mathbf{v}\right|_{m} \leq C h^{s-m}\|\mathbf{v}\|_{s} \quad \forall \mathbf{v} \in\left[H^{s}(\Omega)\right]^{2}
$$

for $m=0$ or 1 and $s=1$ or 2 , with a positive constant $C$ independent of $h$ and $\mathbf{v}$.

To introduce our low order scheme for $(2.7)-(2.9)$, we need to construct discrete bilinear form $a_{h}(\cdot, \cdot)$. We start with constructing local bilinear form $a_{h}^{E}(\cdot, \cdot)$. Specifically, $a_{h}^{E}(\cdot, \cdot)$ is defined by: for all $\mathbf{u}, \mathbf{v} \in \boldsymbol{V}_{\mathrm{BR}}^{E}$

$$
a_{h}^{E}(\mathbf{u}, \mathbf{v}):=2 \mu \tilde{a}_{h}^{E}(\mathbf{u}, \mathbf{v})+\lambda\left(\Pi_{0}^{0, E} \operatorname{div} \mathbf{u}, \Pi_{0}^{0, E} \operatorname{div} \mathbf{v}\right)_{E},
$$

where

$$
\tilde{a}_{h}^{E}(\mathbf{u}, \mathbf{v}):=\left(\Pi_{1}^{0, E} \varepsilon(\mathbf{u}), \Pi_{1}^{0, E} \varepsilon(\mathbf{v})\right)_{E}+S^{E}(\mathbf{u}, \mathbf{v}),
$$

and $S^{E}(\mathbf{u}, \mathbf{v})$ is a stabilizing bilinear form to be defined later. Apparently, for each $\mathbf{v} \in \boldsymbol{V}_{\mathrm{BR}}^{E}$, the following decomposition holds

$$
\mathbf{v}=\mathbf{v}^{1}+\mathbf{v}^{2} \quad \mathbf{v}^{1} \in\left[V_{1}^{E}\right]^{2} \quad \mathbf{v}^{2} \in \operatorname{span}\left\{\boldsymbol{\theta}_{i} 1 \leq i \leq N_{E}\right\} .
$$

Lemma 4.7 implies that the decomposition is unique. For such $\mathbf{v}^{k}(k=1,2)$, we set $\mathbf{v}^{k}=\left(v_{1}^{k}, v_{2}^{k}\right)$. Then the stabilizing term $S^{E}$ can be defined by

$$
S^{E}(\mathbf{u}, \mathbf{v}):=\sum_{k=1}^{2} \sum_{j=1}^{2} \sum_{i=1}^{n_{k}^{E}} \chi_{i}^{k}\left(u_{j}^{k}-\Pi_{k}^{\nabla, E} u_{j}^{k}\right) \chi_{i}^{k}\left(v_{j}^{k}-\Pi_{k}^{\nabla, E} v_{j}^{k}\right),
$$

where $n_{k}^{E}$ is the dimension of $V_{k}^{E}$, and $\chi_{i}^{k}\left(i \in\left\{1,2, \cdots, n_{k}^{E}\right\}\right)$ are the degrees of freedom given by $\widetilde{\mathbf{D}}_{\mathbf{V}} \mathbf{1}-\widetilde{\mathbf{D}}_{\mathbf{V}} \mathbf{3}$. Similar to higher order case, we established the following lemma in [56].

Lemma 4.10. The local discrete bilinear form $\tilde{a}_{h}^{E}(\cdot, \cdot)$ defined by (4.41) satisfies

- consistency: for all $\mathbf{q} \in\left[\mathbb{P}_{1}(E)\right]^{2}$ and $\mathbf{v} \in \boldsymbol{V}_{\mathrm{BR}}^{E}$

$$
\tilde{a}_{h}^{E}(\mathbf{q}, \mathbf{v})=\tilde{a}^{E}(\mathbf{q}, \mathbf{v})
$$

- stability:

$$
\beta_{*}\|\varepsilon(\mathbf{v})\|_{0, E}^{2} \leq \tilde{a}_{h}^{E}(\mathbf{v}, \mathbf{v}) \leq \beta^{*}\|\nabla \mathbf{v}\|_{0, E}^{2} \quad \forall \mathbf{v} \in \boldsymbol{V}_{\mathrm{BR}}^{E}
$$

with positive constants $\beta_{*}$ and $\beta^{*}$ independent of the element $E$. 
We define the global approximated bilinear form $a_{h}(\cdot, \cdot): \boldsymbol{V}_{\mathrm{BR}} \times \boldsymbol{V}_{\mathrm{BR}} \rightarrow \mathbb{R}$ by simply summing the local bilinear forms:

$$
a_{h}(\mathbf{u}, \mathbf{v}):=\sum_{E \in \mathcal{T}_{h}} a_{h}^{E}(\mathbf{u}, \mathbf{v}) \quad \forall \mathbf{u}, \mathbf{v} \in \boldsymbol{V}_{\mathrm{BR}}
$$

We proved in [56] that discrete bilinear form $a_{h}(\cdot, \cdot)$ satisfies the following properties.

Theorem 4.11. The discrete bilinear form $a_{h}(\cdot, \cdot): \boldsymbol{V}_{\mathrm{BR}} \times \boldsymbol{V}_{\mathrm{BR}} \rightarrow \mathbb{R}$ defined by (4.45) satisfies

- boundedness: for all $\mathbf{u}, \mathbf{v} \in \mathbf{V}_{h}$

$$
a_{h}(\mathbf{u}, \mathbf{v}) \leq C_{\lambda}|\mathbf{u}|_{1}|\mathbf{v}|_{1},
$$

with $C_{\lambda}>0$ dependent on $\lambda$

- coercivity: for all $\mathbf{v} \in \mathbf{V}_{h}$

$$
a_{h}(\mathbf{v}, \mathbf{v}) \geq C|\mathbf{v}|_{1}^{2}
$$

with $C>0$ independent of $h$.

We are now in a position to state our low order fully discrete scheme. Given initial data $\mathbf{u}_{h}^{0} \in \boldsymbol{V}_{h}, p_{h}^{0} \in Q_{h}$, the fully discrete scheme of $(2.3)-(2.5)$ seeks $\left(\mathbf{u}_{h}^{n}, \mathbf{w}_{h}^{n}, p_{h}^{n}\right) \in \boldsymbol{V}_{h} \times \boldsymbol{\Sigma}_{h} \times Q_{h}$ at time $t_{n}, 1 \leq n \leq N$, such that

$$
\begin{aligned}
a_{h}\left(\mathbf{u}_{h}^{n}, \mathbf{v}_{h}\right)-\alpha\left(\operatorname{div} \mathbf{v}_{h}, p_{h}^{n}\right) & =\left(\mathbf{f}_{h}^{n}, \mathbf{v}_{h}\right), & & \forall \mathbf{v}_{h} \in \boldsymbol{V}_{h}, \\
m_{h}\left(\mathbf{w}_{h}^{n}, \mathbf{z}_{h}\right)-\left(\operatorname{div} \mathbf{z}_{h}, p_{h}^{n}\right) & =0, & & \forall \mathbf{z}_{h} \in \mathbf{\Sigma}_{h}, \\
c_{0}\left(\frac{p_{h}^{n}-p_{h}^{n-1}}{\Delta t}, q_{h}\right)+\alpha\left(\operatorname{div}\left(\frac{\mathbf{u}_{h}^{n}-\mathbf{u}_{h}^{n-1}}{\Delta t}\right), q_{h}\right)+\left(\operatorname{div}_{h}^{n}, q_{h}\right) & =\left(g^{n}, q_{h}\right), & & \forall q_{h} \in Q_{h} .
\end{aligned}
$$

Using the same argument as in the higher order case with the help of Theorem 4.11, we can prove that at each time step $t_{n}$, the above system is well-posed.

Remark 4.12. Elliptic projection is defined similarly as (4.4)-(4.6). Thanks to Theorem 4.9, we can get similar result as in (4.7)-(4.9). Then initial data $\mathbf{u}_{h}^{0} \in \boldsymbol{V}_{h}, p_{h}^{0} \in Q_{h}$ can be constructed as (4.11).

To derive optimal error estimates in low order case, we need the following regularity assumptions:

$$
\begin{aligned}
\mathbf{u} & \in L^{\infty}\left(\left[0, T_{0}\right] ; H^{2}(\Omega)\right), & & \mathbf{f}, \lambda \operatorname{div} \mathbf{u}, \mathbf{w}, p \in L^{\infty}\left(\left[0, T_{0}\right] ; H^{1}(\Omega)\right), \\
\mathbf{u}_{t} & \in L^{2}\left(\left[0, T_{0}\right] ; H^{2}(\Omega)\right), & & \mathbf{f}_{t}, \lambda \operatorname{div} \mathbf{u}_{t}, \mathbf{w}_{t}, p_{t} \in L^{2}\left(\left[0, T_{0}\right] ; H^{1}(\Omega)\right), \\
\mathbf{u}_{t t} & \in L^{2}\left(\left[0, T_{0}\right] ; H^{1}(\Omega)\right), & & \mathbf{f}_{t t} \in L^{2}\left(\left[0, T_{0}\right] ; L^{2}(\Omega)\right) .
\end{aligned}
$$

With the help of Theorem 4.9 and Lemma 4.10, we can prove the following theorem by using the same argument as in the proof of Theorem 4.3 .

Theorem 4.13. Let $(\mathbf{u}, \mathbf{w}, p)$ be the solution of (2.7)-(2.9) and $\left(\mathbf{u}_{h}^{n}, \mathbf{w}_{h}^{n}, p_{h}^{n}\right)$ the solution of fully discrete problem (4.48)-(4.50). Assume that (4.51) holds. Then we have the following error estimates:

$$
\max _{1 \leq n \leq N}\left(\left\|\mathbf{u}^{n}-\mathbf{u}_{h}^{n}\right\|_{1}\right)^{2}+\Delta t \sum_{n=0}^{N}\left(\left\|\mathbf{w}^{n}-\mathbf{w}_{h}^{n}\right\|_{0}^{2}+\left\|p^{n}-p_{h}^{n}\right\|_{0}^{2}\right) \leq C\left(h^{2}+\Delta t^{2}\right),
$$

where $C$ is a positive constant independent of $h$ and $\lambda$. 


\section{Numerical tests}

In this section, we provide three numerical tests to validate the theoretical results proposed in the previous sections. The first test is used to show that our methods can overcome Poisson locking (when $\lambda \rightarrow \infty$ ). The last two tests are devoted to validate the effectiveness of our methods to eliminate pressure oscillation (when $c_{0}=0$ and $\mathbf{K} \approx 0$ ). In all tests, the algorithms are implemented by using the MATLAB. The implementation aspects of virtual element method can be found in $[10,12,55]$. We will solve the problems using the following methods:

$$
\begin{aligned}
& \text { Method 1: } \boldsymbol{V}_{h}:=\left[V_{1}\right]^{2} \cap \boldsymbol{V}, \boldsymbol{\Sigma}_{h}:=\Sigma_{h}^{0} \cap \boldsymbol{\Sigma}, Q_{h}:=\mathbb{P}_{0}\left(\mathcal{T}_{h}\right), \\
& \text { Method 2: } \boldsymbol{V}_{h}:=\left[V_{2}\right]^{2} \cap \boldsymbol{V}, \boldsymbol{\Sigma}_{h}:=\Sigma_{h}^{1} \cap \boldsymbol{\Sigma}, Q_{h}:=\mathbb{P}_{1}\left(\mathcal{T}_{h}\right), \\
& \text { Method 3: } \boldsymbol{V}_{h}:=\boldsymbol{V}_{\mathrm{BR}} \cap \boldsymbol{V}, \boldsymbol{\Sigma}_{h}:=\Sigma_{h}^{0} \cap \boldsymbol{\Sigma}, Q_{h}:=\mathbb{P}_{0}\left(\mathcal{T}_{h}\right) .
\end{aligned}
$$

\subsection{Accuracy for a smooth solution with a large $\lambda$}

We start with considering a test with analytical solution [62]. This test is devoted to confirm the optimal convergence rates predicted in Theorems 4.3 and 4.13. The body force $\mathbf{f}$ and source/sink term $g$ are chosen so that the exact solution on the computational domain $\Omega=(0,1)^{2}$ is

$$
\begin{aligned}
u_{1} & =e^{-t} \sin (2 \pi y)(-1+\cos (2 \pi x))+1 /(\mu+\lambda) \sin (\pi x) \sin (\pi y), \\
u_{2} & =e^{-t} \sin (2 \pi x)(1-\cos (2 \pi y))+1 /(\mu+\lambda) \sin (\pi x) \sin (\pi y), \\
p & =e^{-t} \sin (\pi x) \sin (\pi y) .
\end{aligned}
$$

Dirichlet boundary conditions are imposed for $\mathbf{u}$ and $p$ according to the exact solution. Note that $\operatorname{div} \mathbf{u}=\pi e^{-t} \sin (\pi(x+y)) /(\mu+\lambda) \rightarrow 0$ as $\lambda \rightarrow \infty$ for any time $t$. Therefore, the exact solution is suitable to test whether the proposed methods can overcome Poisson locking. Following [62], we choose the following material parameters:

$$
c_{0}=0, \quad \alpha=1, \quad \mathbf{K}=1, \quad \lambda=10^{4}, \quad \mu=1 .
$$

In order to compute the VEM errors, we consider the computable error quantities:

$$
\begin{aligned}
& \boldsymbol{E}_{u}=\max _{1 \leq n \leq N}\left(\left(\sum_{E \in \mathcal{T}_{h}}\left\|\mathbf{u}^{n}-\Pi_{k}^{0, E} \mathbf{u}_{h}^{n}\right\|_{0, E}^{2}+\left\|\nabla \mathbf{u}^{n}-\Pi_{k-1}^{0, E} \nabla \mathbf{u}_{h}^{n}\right\|_{0, E}^{2}\right)^{1 / 2}\right) \\
& \boldsymbol{E}_{w}=\sum_{n=0}^{N}\left\|\mathbf{w}^{n}-\Pi_{k}^{0} \mathbf{w}_{h}^{n}\right\|_{0}^{2} \Delta t \\
& \boldsymbol{E}_{p}=\sum_{n=0}^{N}\left\|p^{n}-p_{h}^{n}\right\|_{0}^{2} \Delta t
\end{aligned}
$$

In this test, the square domain is partitioned using the following sequences of polygonal meshes:

- $\left\{\mathcal{T}_{h}^{1}\right\}_{h}$ : triangular meshes with $h=1 / 8,1 / 16,1 / 32,1 / 64$,

- $\left\{\mathcal{T}_{h}^{2}\right\}_{h}:$ quadrilateral meshes with $h=1 / 8,1 / 16,1 / 32,1 / 64$,

- $\left\{\mathcal{T}_{h}^{3}\right\}_{h}$ : non-convex cells with $h=1 / 5,1 / 10,1 / 20,1 / 40$.

An example of the meshes is shown in Figure 1. For Methods 1 and 3, we take the backward Euler scheme with $\Delta t=T_{0} /(\operatorname{ceil}(1 / h))$ for time discretization, where ceil $(X)$ rounds the elements of $X$ to the nearest integers towards infinity. In order to make the spatial direction error not affected by the time direction error, we use Backward Difference Formula of second order (BDF2) for Method 2. It can be proved that related errors are scale as $\Delta t^{2}+h^{2}$, but the detailed proof will be omitted for simplicity.

Figures 2-4 display convergence results for the various mesh families and different methods. The results of Methods 1 and 3 are in very good agreement with the convergence rates predicted in previous sections. 

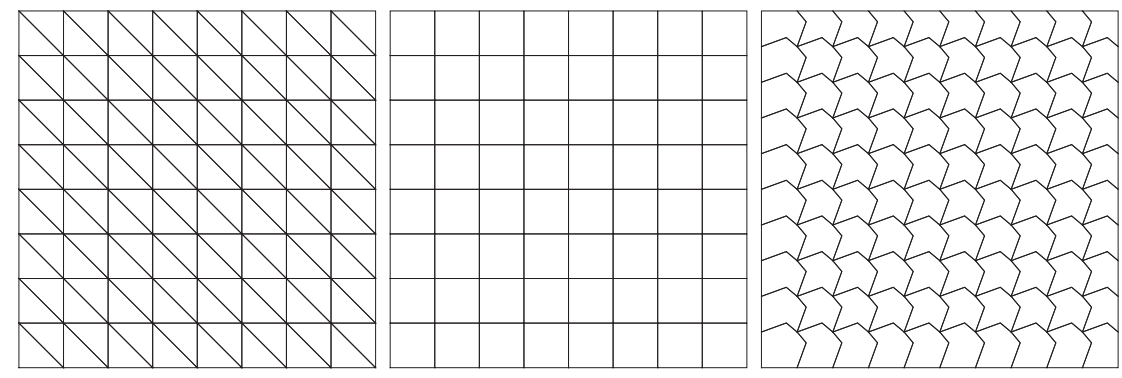

FIGURE 1. Example of the adopted polygonal meshes: $\mathcal{T}_{1 / 8}^{1}, \mathcal{T}_{1 / 8}^{2}, \mathcal{T}_{1 / 10}^{3}$.

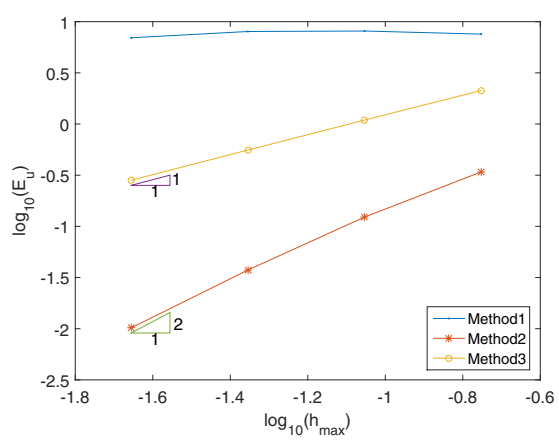

(a)

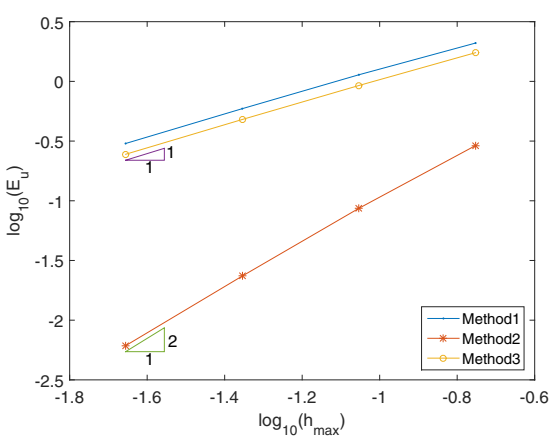

(b)

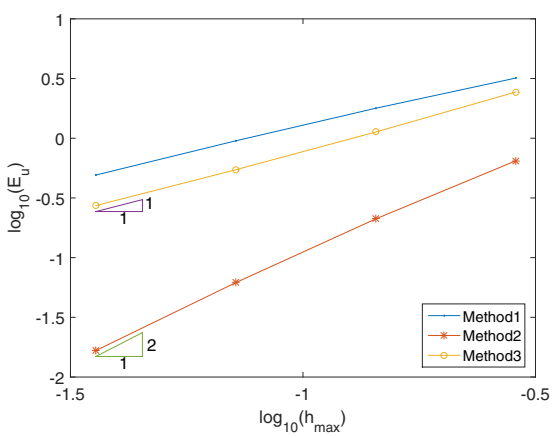

(c)

Figure 2. $E_{u}$ on different meshes. (a) Triangular; (b) quadrilateral; (c) non-convex.

As predicted in Theorems 4.3 and 4.13, the errors are robust to $\lambda$. From Figure 2a, we can see that the Poisson locking occurs in the triangular mesh if we use Method 1. However, this can be overcome if the Method 2 or Method 3 are used in the triangular meshes. An interesting thing is that Method 1 does not suffer the Poisson locking in the quadrilateral and non-convex meshes. Moreover it reaches the optimal convergence rate. It seems that for the method 1, the discrete inf-sup condition relies on mesh types. This requires further study. Although errors $\boldsymbol{E}_{u}$ of Methods 1 and 3 converge with optimal rate 1, Method 3 is more accurate than Method 1 according to Figures 2b and 2c. Figures 3 and 4 show that convergence curves of Methods 1 and 3 are almost the same, which means that in this test case, Method 1 still yields the optimal rates for the pressure and flux variables, even though its displacement variable is locking in triangular meshes. Overall, Figures $2-4$ confirm the theoretical results in Theorems 4.3 and 4.13 . 


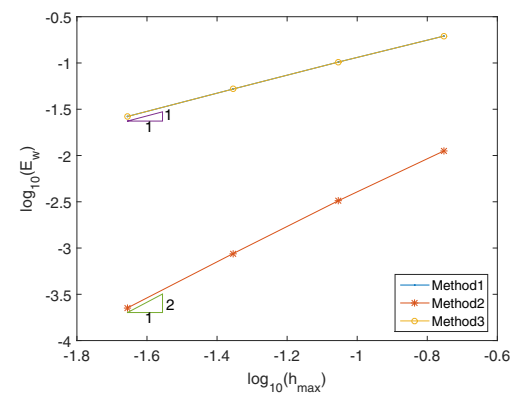

(a)

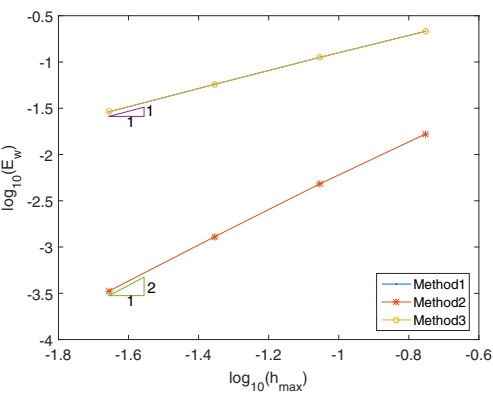

(b)

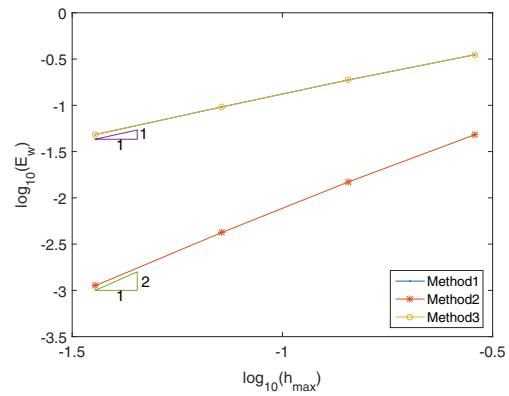

(c)

Figure 3. $E_{w}$ on different meshes. (a) Triangular; (b) quadrilateral; (c) non-convex.

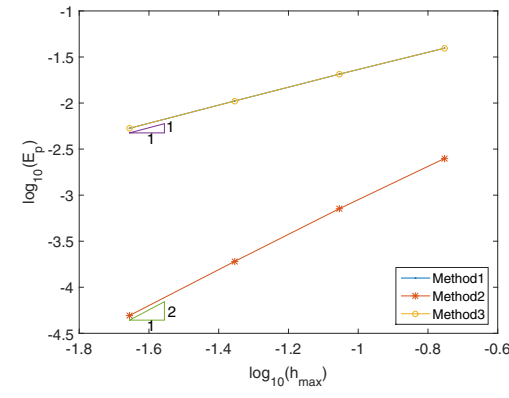

(a)

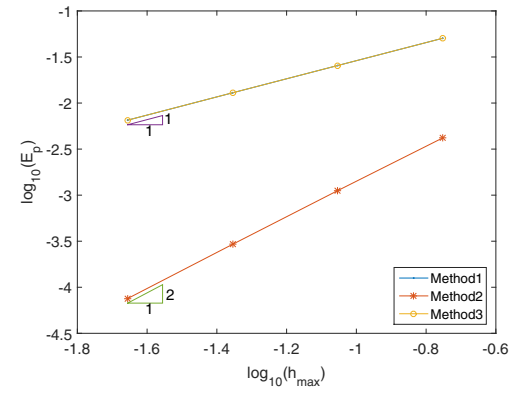

(b)

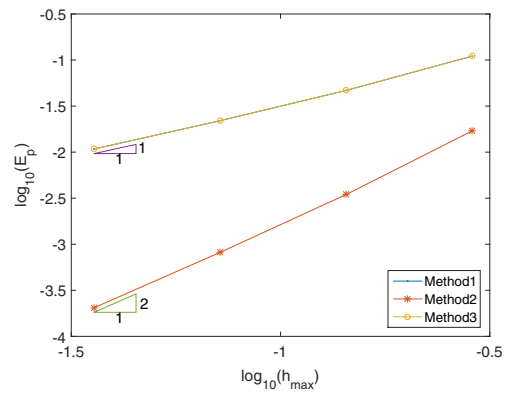

(c)

Figure 4. $E_{p}$ on different meshes. (a) Triangular; (b) quadrilateral; (c) non-convex. 


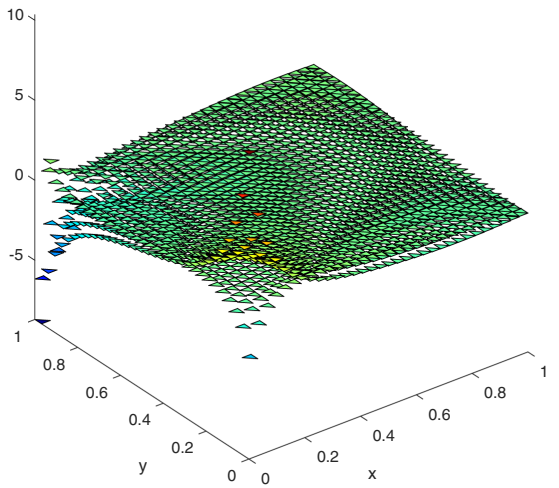

(a)

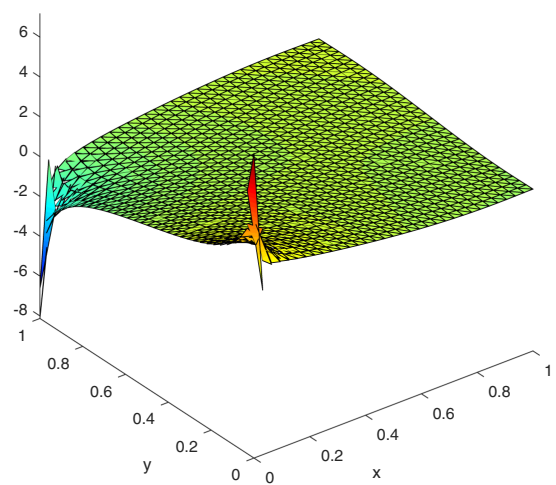

(b)

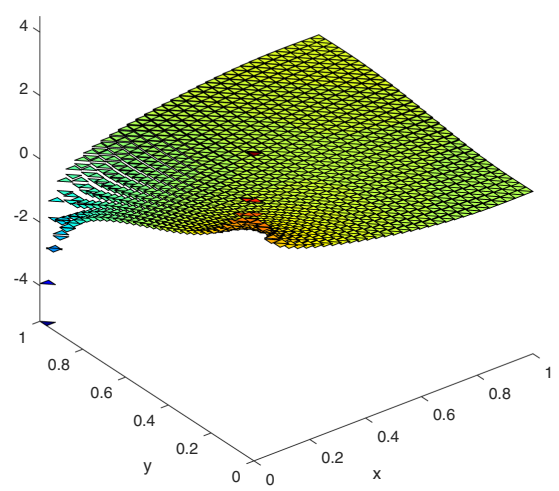

(c)

FIgURE 5. Numerical pressure for the cantilever bracket problem on $\mathcal{T}_{1 / 32}^{1}$ at time $t=0.001$. (a) Method 1; (b) Method 2; (c) Method 3.

\subsection{Cantilever bracket problem}

We now consider a cantilever bracket problem $[48,52]$. The computational domain is again $\Omega=(0,1)^{2}$. For the elasticity problem, a no-displacement boundary condition is imposed in the left side edge. We also impose a downward traction at top side and a traction-free boundary condition at the right and bottom sides. The initial displacement and pressure are assumed to be zero. The material parameters are set as follows:

$$
\alpha=0.93, \quad c_{0}=0, \quad \mathbf{K}=10^{-7}, \quad E=10^{5}, \quad \nu=0.4,
$$

where $E$ and $\nu$ denote Young's modulus and the Poisson ratio, respectively, and there hold

$$
\mu=\frac{E}{2(1+\nu)}, \quad \lambda=\frac{E \nu}{(1-2 \nu)(1+\nu)} .
$$

Figures 5-7 present a comparison of the numerical pressure after one time step $\Delta t=0.001$ using three different methods on three different types of meshes. It can be seen from Figure 5 that pressure obtained by Method 1 has spurious oscillations that are mostly concentrated to the points $(0,0)$ and $(0,1)$. By contrast, the pressure computed by Methods 2 and 3 does not occur such oscillations, no matter what type of mesh is used. We observe from Figures 6 and 7 that Method 1 does not produce pressure oscillations on quadrilateral and non-convex meshes. 


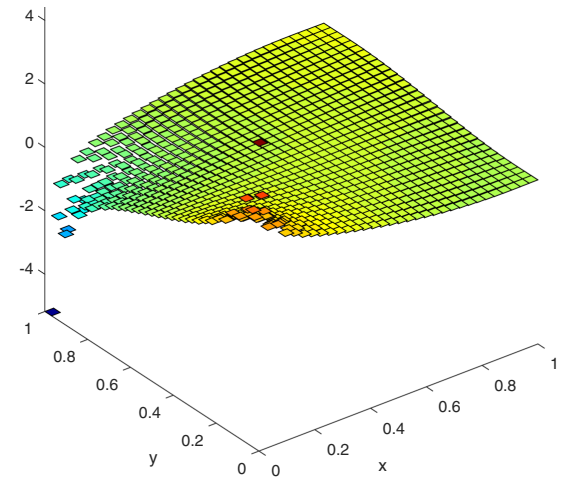

(a)

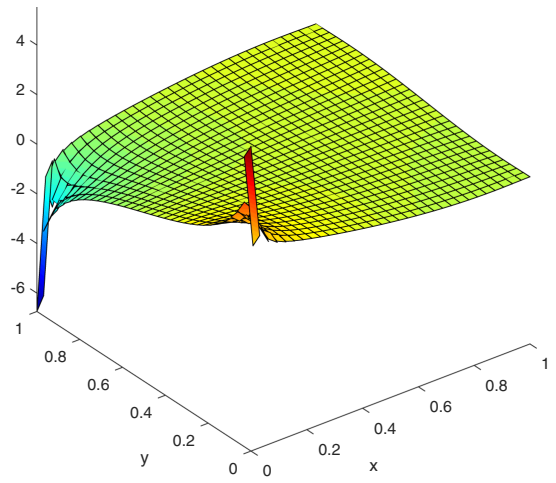

(b)

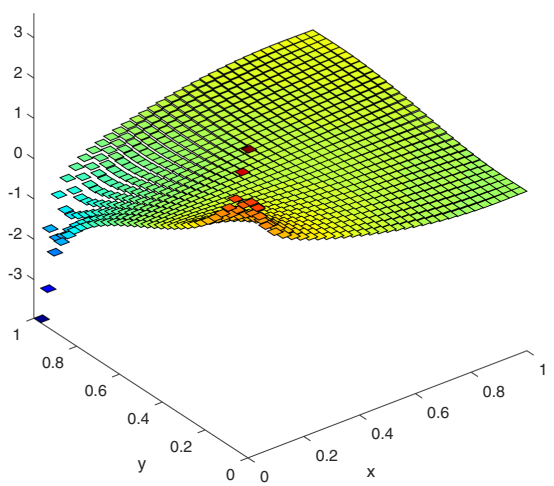

(c)

FiguRE 6. Numerical pressure for the cantilever bracket problem on $\mathcal{T}_{1 / 32}^{2}$ at time $t=0.001$. (a) Method 1; (b) Method 2; (c) Method 3.

\subsection{Barry and Mercer's problem}

We now consider another bench mark test called Barry and Mercer's problem [6]. It models the behavior of a rectangular uniform porous material with a pulsating point source under a specific set of boundary conditions. More precisely, we let $\Omega=(0,1)^{2}$ and consider the following boundary conditions on $\partial \Omega$

$$
\mathbf{u} \cdot \boldsymbol{t}=0, \quad \boldsymbol{n}^{T} \nabla \mathbf{u} \boldsymbol{n}=0, \quad p=0,
$$

where $\boldsymbol{t}$ and $\boldsymbol{n}$ denote the tangent vector and outward normal vector on $\partial \Omega$, respectively. For this problem, we choose the following material parameters:

$$
\alpha=1, \quad c_{0}=0, \quad E=10^{5}, \quad \nu=0.1, \quad \mathbf{K}=10^{-2} .
$$

The evolution of the displacement and pressure fields is driven by a periodic pointwise source defined by

$$
g=\delta\left(\boldsymbol{x}-\boldsymbol{x}_{0}\right) \sin (\hat{t}),
$$

where $\boldsymbol{x}_{0}=(0.25,0.25), \hat{t}=\beta t$ and $\beta:=(\lambda+2 \mu) \mathbf{K}$.

Figure 8 displays the numerical pressure computed by Method 3 on quadrilateral mesh $\mathcal{T}_{1 / 64}^{2}$ at normalized times $\hat{t}=\pi / 2$ and $\hat{t}=3 \pi / 2$. We also plot the deformed domain according to the results obtained by the 


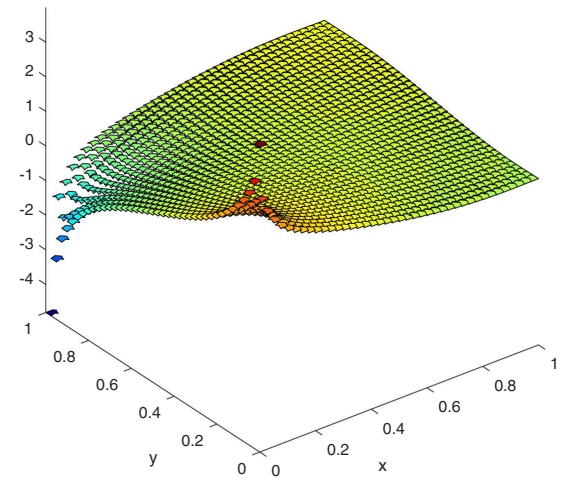

(a)

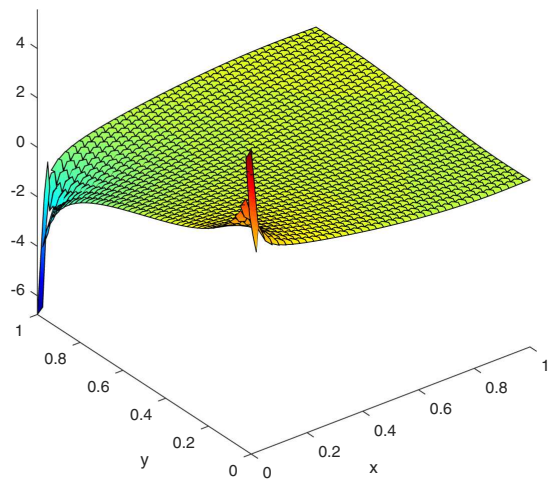

(b)

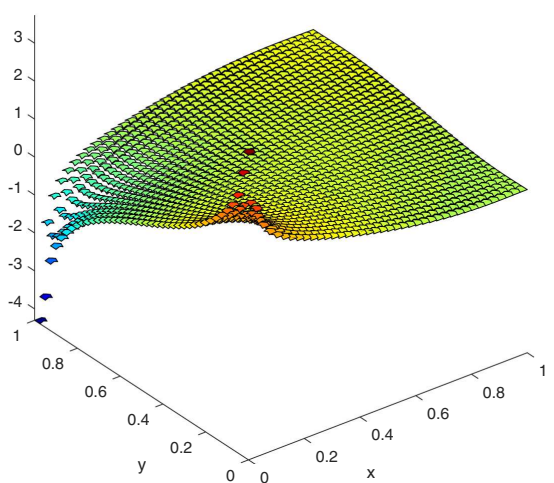

(c)

FiguRE 7. Numerical pressure for the cantilever bracket problem on $\mathcal{T}_{1 / 40}^{3}$ at time $t=0.001$. (a) Method 1; (b) Method 2; (c) Method 3.

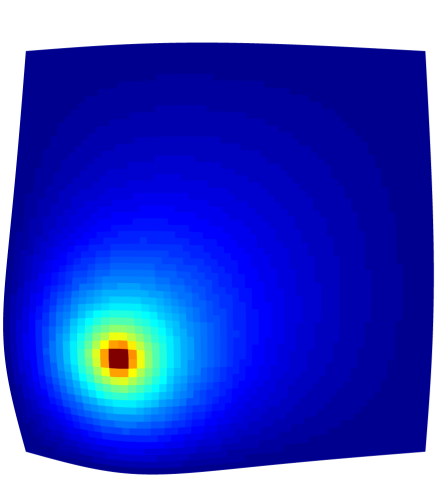

(a)
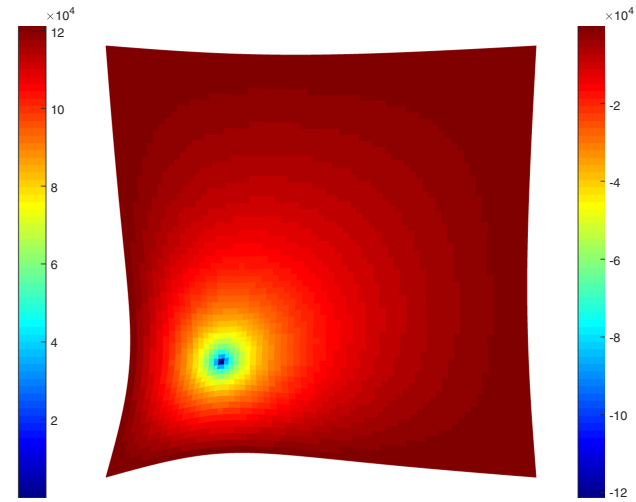

(b)

FiguRE 8. Numerical pressure on the deformed domain at different times for quadrilateral meshes with $h=1 / 64$ using Method 3. (a) $\hat{t}=\pi / 2$; (b) $\hat{t}=3 \pi / 2$. 


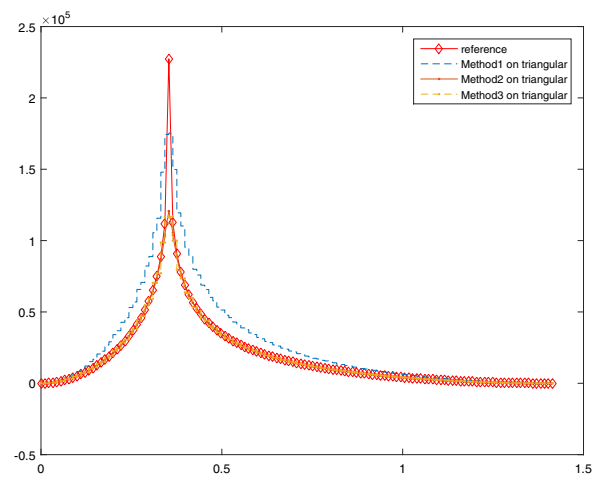

(a)

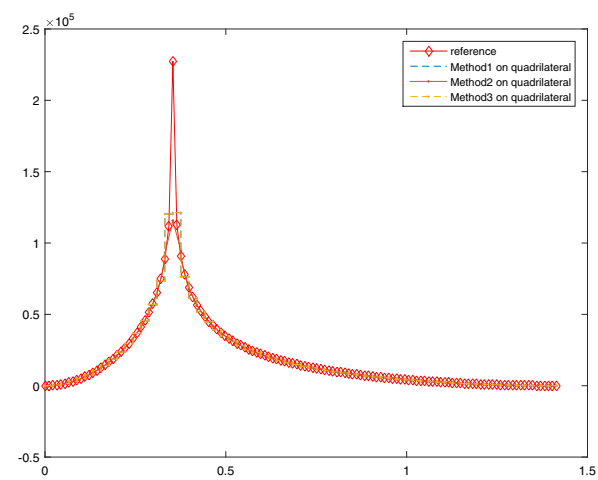

(c)

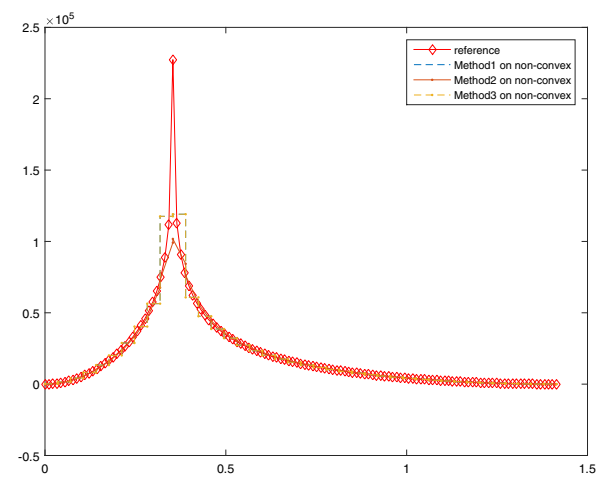

(e)

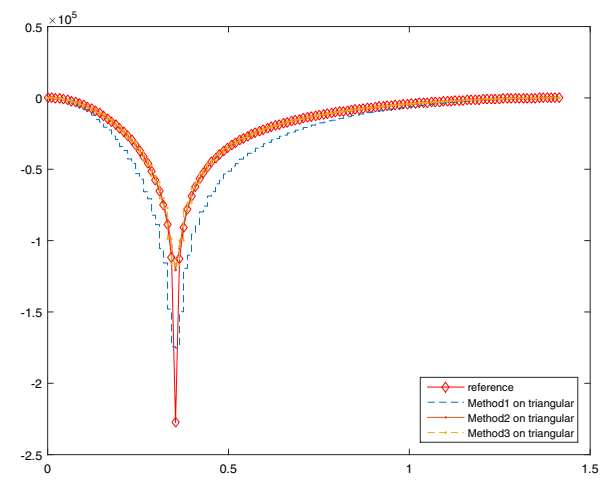

(b)

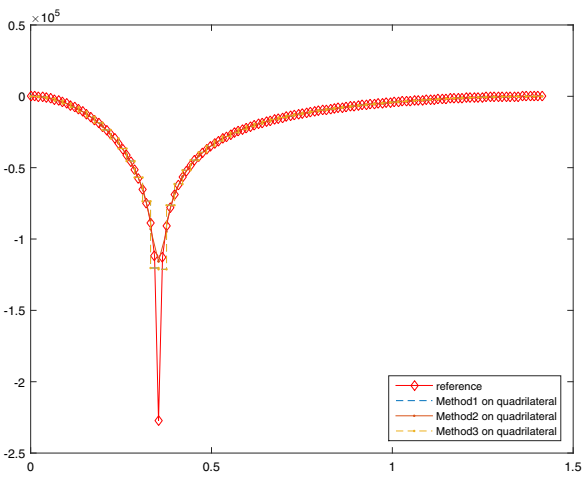

(d)

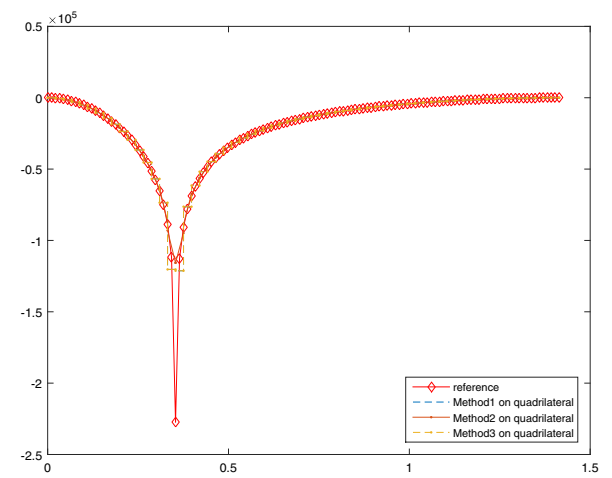

(f)

Figure 9. Numerical pressure computed by Methods 1-3 along the diagonal $(0,0)-(1,1)$ of the domain for different time on different meshes. (a) $\hat{t}=\pi / 2$, triangular mesh; (b) $\hat{t}=3 \pi / 2$, triangular mesh; (c) $\hat{t}=\pi / 2$, quadrilateral mesh; (d) $\hat{t}=3 \pi / 2$, quadrilateral mesh; (e) $\hat{t}=\pi / 2$, non-convex mesh; (f) $\hat{t}=3 \pi / 2$, non-convex mesh. 


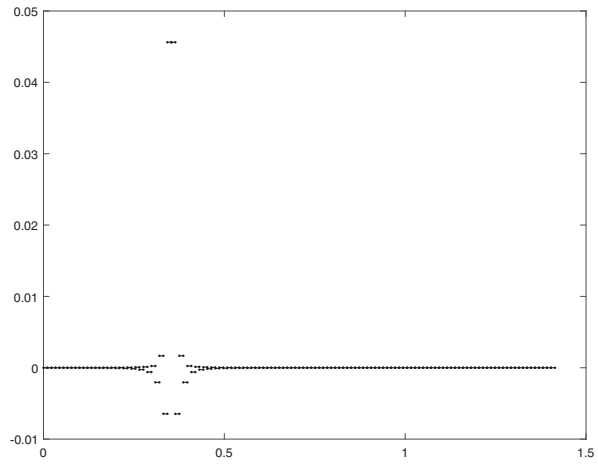

(a)

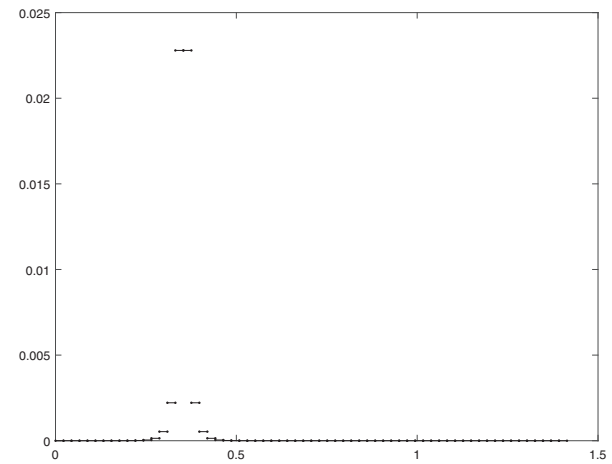

(c)

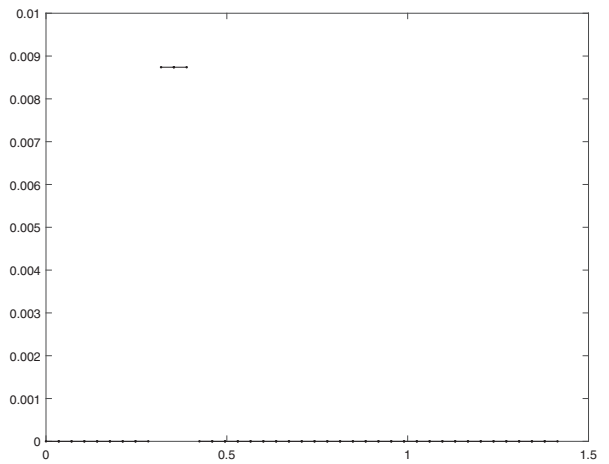

(e)

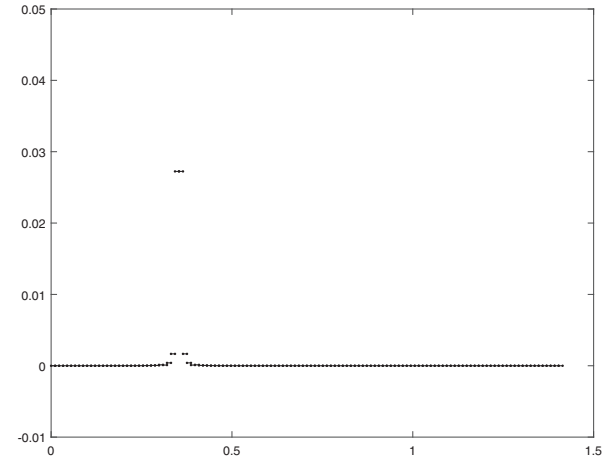

(b)

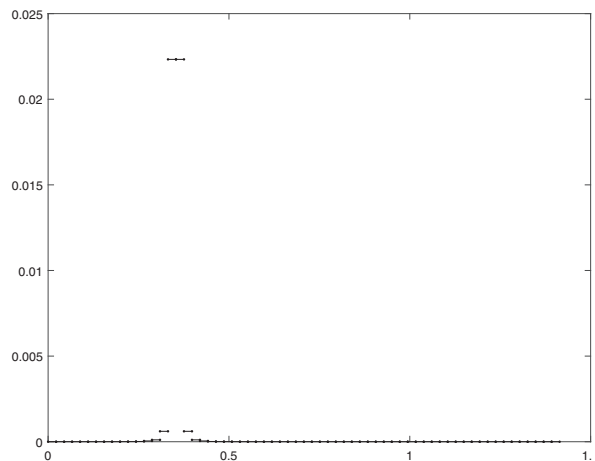

(d)

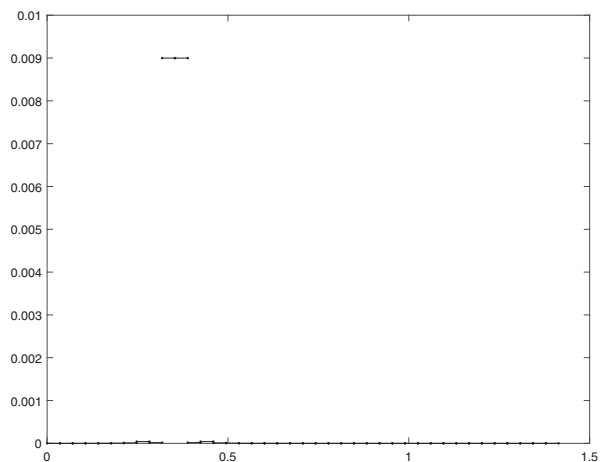

(f)

Figure 10. Numerical pressure computed by Methods 1 and 3 along the diagonal $(0,0)-(1,1)$ of the domain for $\mathbf{K}=10^{-6}$ and time step $\Delta t=10^{-4}$ on different meshes. (a) Method 1 on triangular mesh; (b) Method 3 on triangular mesh; (c) Method 1 on quadrilateral mesh; (d) Method 3 on quadrilateral mesh; (e) Method 1 on non-convex mesh; (f) Method 3 on non-convex mesh. 
displacements. We observe that at $\hat{t}=\pi / 2$, the source is positive, and this fluid injection causes an inflation of the poroelastic medium. On the other hand, we can see a contraction of the medium since the source term is negative at $\hat{t}=3 \pi / 2$. We also plot the pressure profiles computed by Methods $1-3$ on three different types of meshes at normalized times $\hat{t}=\pi / 2$ and $\hat{t}=3 \pi / 2$ with the analytical solution along the diagonal $(0,0)-(1,1)$ of the domain in Figure 9. The analytical solution of this problem is given by infinite series, which can be found in $[6,48]$. In all the cases, a time step $\Delta t=2 \pi / \beta \cdot 10^{-2}$ is used. From Figure 9, we can see that all the numerical pressures can capture the behaviour of the exact solution except the one computed by Method 1 on triangular mesh. Probably, this is because Theorem 3.7 does not hold for $k=1$, so according to [62] this may cause pressure oscillation when $c_{0}=0$ and $\mathbf{K} \approx 0$.

To further check the robustness of Method 3 with respect to pressure oscillations for small permeability combined with small time step, we also show in Figure 10 the numerical pressure obtained by Methods 1 and 3 with $\mathbf{K}=10^{-6}$ and $\Delta=10^{-4}$ on three different types of meshes. It is clear from Figure 10a that Method 1 on triangular mesh produced nonphysical oscillations in the pressure variable. By contrast, we do not observe significant oscillation from Figure 10b, that is to say Method 3 does eliminate the pressure oscillation. From Figures 10c-10f, we observe that Method 1 does not produce pressure oscillation on quadrilateral and nonconvex meshes, and the numerical pressure of Method 1 resembles the one of Method 3 on these meshes. We infer that Theorem 3.7 may hold on quadrilateral and non-convex meshes, which needs further research.

\section{Conclusion}

In this paper, we propose and analyze virtual element methods for Biot's consolidation model. One is a high-order scheme, and the other is a low-order scheme. We show the a priori error estimates of fully discrete problems. In particular, our error estimates hold when the specific storage coefficient vanishes, and the generic constants in error estimates are uniformly bounded with respect to Lamé constant $\lambda$. Therefore, our methods not only can overcome Poisson locking but also does not suffer pressure oscillations. Numerical tests illustrate the validity of our theoretical analysis.

Acknowledgements. The authors would like to thank the editors and referees for their useful and constructive comments that helped us to improve this paper. This research is supported by the Open Fund (PLN201808) of State Key Laboratory of Oil and Gas Reservoir Geology and Exploitation (Southwest Petroleum University). This research is supported by the National Natural Science Foundation of China (No. 11971337).

\section{REFERENCES}

[1] R. Adams and J. Fournier, Sobolev Spaces. Vol. 140 of Pure and Applied Mathematics. Academic Press (2003).

[2] B. Ahmad, A. Alsaedi, F. Brezzi, L.D. Marini and A. Russo, Equivalent projectors for virtual element methods. Comput. Math. Appl. 66 (2013) 376-391.

[3] P.F. Antonietti, L. Beirão da Veiga, D. Mora and M. Verani, A stream virtual element formulation of the Stokes problem on polygonal meshes. SIAM J. Numer. Anal. 52 (2014) 386-404.

[4] P.F. Antonietti, L. Beirão da Veiga, S. Scacchi and M. Verani, A $C^{1}$ virtual element method for the Cahn-Hilliard equation with polygonal meshes. SIAM J. Numer. Anal. 54 (2016) 34-56.

[5] E. Artioli, S. de Miranda, C. Lovadina and L. Patruno, A stress/displacement virtual element method for plane elasticity problems. Comput. Methods Appl. Mech. Eng. 325 (2017) 155-174.

[6] S.I. Barry and G.N. Mercer, Exact solutions for two-dimensional time-dependent flow and deformation within a poroelastic medium. Trans. ASME J. Appl. Mech. 6 (1999) 536-540.

[7] L. Beirão da Veiga and K. Lipnikov, A mimetic discretization of the Stokes problem with selected edge bubbles. SIAM J. Sci. Comput. 32 (2010) 875-893.

[8] L. Beirão da Veiga, F. Brezzi and L.D. Marini, Virtual elements for linear elasticity problems. SIAM J. Numer. Anal. 51 (2013) 794-812.

[9] L. Beirão da Veiga, F. Brezzi, A. Cangiani, G. Manzini, L.D. Marini and A. Russo, Basic principles of virtual element methods. Math. Models Methods Appl. Sci. 23 (2013) 199-214.

[10] L. Beirão da Veiga, F. Brezzi, L.D. Marini and A. Russo, The hitchhiker's guide to the virtual element method. Math. Models Methods Appl. Sci. 24 (2014) 1541-1573. 
[11] L. Beirão da Veiga, K. Lipnikov and G. Manzini, The Mimetic Finite Difference Method for Elliptic Problems. Vol. 11 of MSESA - Modeling, Simulation and Applications. Springer, Cham (2014).

[12] L. Beirão da Veiga, F. Brezzi, L.D. Marini and A. Russo, Virtual Element Implementation for General Elliptic Equations. Springer International Publishing, Cham (2016) 39-71.

[13] L. Beirão da Veiga, F. Brezzi, L.D. Marini and A. Russo, Virtual element method for general second-order elliptic problems on polygonal meshes. Math. Models Methods Appl. Sci. 26 (2016) 729-750.

[14] L. Beirão da Veiga, F. Brezzi, L.D. Marini and A. Russo, Mixed virtual element methods for general second order elliptic problems on polygonal meshes. ESAIM: M2AN 50 (2016) 727-747.

[15] L. Beirão da Veiga, C. Lovadina and A. Russo, Stability analysis for the virtual element method. Math. Models Methods Appl. Sci. 27 (2017) 2557-2594.

[16] L. Beirão da Veiga, C. Lovadina and G. Vacca, Divergence free virtual elements for the Stokes problem on polygonal meshes. ESAIM: M2AN 51 (2017) 509-535.

[17] L. Beirão da Veiga, C. Lovadina and G. Vacca, Virtual elements for the Navier-Stokes problem on polygonal meshes. SIAM J. Numer. Anal. 56 (2018) 1210-1242.

[18] M.F. Benedetto, S. Berrone, A. Borio, S. Pieraccini and S. Scialò, Order preserving SUPG stabilization for the virtual element formulation of advection-diffusion problems. Comput. Methods Appl. Mech. Eng. 311 (2016) 18-40.

[19] L. Berger, R. Bordas, D. Kay and S. Tavener, Stabilized lowest-order finite element approximation for linear three-field poroelasticity. SIAM J. Sci. Comput. 37 (2015) A2222-A2245.

[20] C. Bernardi and G. Raugel, Analysis of some finite elements for the Stokes problem. Math. Comput. 44 (1985) $71-79$.

[21] M.A. Biot, General theory of three-dimensional consolidation. J. Appl. Phys. 12 (1941) 155-164.

[22] M.A. Biot, Theory of elasticity and consolidation for a porous anisotropic solid. J. Appl. Phys. 26 (1955) $182-185$.

[23] D. Boffi, M. Botti and D.A. Di Pietro, A nonconforming high-order method for the Biot problem on general meshes. SIAM J. Sci. Comput. 38 (2016) A1508-A1537.

[24] S.C. Brenner and L.R. Scott, The Mathematical Theory of Finite Element Methods. Vol. 15 of Texts in Applied Mathematics. Springer, New York (2007).

[25] S.C. Brenner, Q. Guan and L. Sung, Some estimates for virtual element methods. Comput. Methods Appl. Math. 17 (2017) $553-574$.

[26] F. Brezzi, R.S. Falk and L.D. Marini, Basic principles of mixed virtual element methods. ESAIM: M2AN 48 (2014) $1227-1240$.

[27] R. Bürger, S. Kumar, D. Mora, R. Ruiz-Baier and N. Verma, Virtual element methods for the three-field formulation of time-dependent linear poroelasticity. Preprint arXiv:1912.06029.

[28] A. Cangiani, E.H. Georgoulis, T. Pryer and O.J. Sutton, A posteriori estimates for the virtual element method. Numer. Math. 137 (2017) 857-893.

[29] O. Čertík, F. Gardini, G. Manzini and G. Vacca, The virtual element method for eigenvalue problems with potential terms on polytopic meshes. Appl. Math. 63 (2018) 333-365.

[30] G. Chen and M. Feng, Stabilized finite element methods for Biot's consolidation problems using equal order elements. Adv. Appl. Math. Mech. 10 (2018) 77-99.

[31] L. Chen and J. Huang, Some error analysis on virtual element methods. Calcolo 55 (2018) 5.

[32] Y. Chen, Y. Luo and M. Feng, Analysis of a discontinuous Galerkin method for the Biot's consolidation problem. Appl. Math. Comput. 219 (2013) 9043-9056.

[33] Y. Chen, G. Chen and X. Xie, Weak Galerkin finite element method for Biot's consolidation problem. J. Comput. Appl. Math. 330 (2018) 398-416.

[34] J. Coulet, I. Faille, V. Girault, N. Guy and F. Nataf, Fully coupled schemes using virtual element and finite volume discretisations for Biot equations modelling. In: ECMOR XVI-16th European Conference on the Mathematics of Oil Recovery (2018).

[35] M. Fortin, Old and new finite elements for incompressible flows. Int. J. Numer. Methods Fluids 1 (1981) 347-364.

[36] G. Fu, A high-order HDG method for the Biot's consolidation model. Comput. Math. Appl. 77 (2019) $237-252$.

[37] A.L. Gain, C. Talischi and G.H. Paulino, On the virtual element method for three-dimensional linear elasticity problems on arbitrary polyhedral meshes. Comput. Methods Appl. Mech. Eng. 282 (2014) 132-160.

[38] F. Gardini and G. Vacca, Virtual element method for second-order elliptic eigenvalue problems. IMA J. Numer. Anal. 38 (2018) 2026-2054.

[39] F. Gardini, G. Manzini and G. Vacca, The nonconforming Virtual Element Method for eigenvalue problems. ESAIM: M2AN 53 (2019) 749-774.

[40] X. Hu, C. Rodrigo, F.J. Gaspar and L.T. Zikatanov, A nonconforming finite element method for the Biot's consolidation model in poroelasticity. J. Comput. Appl. Math. 310 (2017) 143-154.

[41] X. Hu, L. Mu and X. Ye, Weak Galerkin method for the Biot's consolidation model. Comput. Math. Appl. 75 (2018) $2017-2030$.

[42] J.J. Lee, Robust three-field finite element methods for Biot's consolidation model in poroelasticity. BIT 58 (2018) $347-372$.

[43] X. Liu and Z. Chen, The nonconforming virtual element method for the Navier-Stokes equations. Adv. Comput. Math. 45 (2019) 51-74.

[44] D. Mora, G. Rivera and R. Rodríguez, A virtual element method for the Steklov eigenvalue problem. Math. Models Methods Appl. Sci. 25 (2015) 1421-1445.

[45] M. Murad and A. Loula, Improved accuracy in finite element analysis of Biot's consolidation problem. Comput. Methods Appl. Mech. Eng. 95 (1992) 359-382. 
[46] M. Murad and A. Loula, On stability and convergence of finite element approximations of Biot's consolidation problem. Int. J. Numer. Methods Eng. 37 (1994) 645-667.

[47] M. Murad, V. Thomée and A. Loula, Asymptotic behavior of semidiscrete finite-element approximations of Biot's consolidation problem. SIAM J. Numer. Anal. 33 (1996) 1065-1083.

[48] P.J. Phillips, Finite element methods in linear poroelasticity: theoretical and computational results, Ph.D. thesis. ProQuest LLC, Ann Arbor, MI/The University of Texas, Austin (2005).

[49] P.J. Phillips and M.F. Wheeler, A coupling of mixed and continuous Galerkin finite element methods for poroelasticity I: the continuous in time case. Comput. Geosci. 11 (2007) 131-144.

[50] P.J. Phillips and M.F. Wheeler, A coupling of mixed and continuous Galerkin finite element methods for poroelasticity II: the discrete in time case. Comput. Geosci. 11 (2007) 145-158.

[51] P.J. Phillips and M.F. Wheeler, A coupling of mixed and discontinuous Galerkin finite element methods for poroelasticity. Comput. Geosci. 12 (2008) 417-435.

[52] P.J. Phillips and M.F. Wheeler, Overcoming the problem of locking in linear elasticity and poroelasticity: an heuristic approach. Comput. Geosci. 13 (2009) 5-12.

[53] M.B. Reed, An investigation of numerical errors in the analysis of consolidation by finite elements. Int. J. Numer. Anal. 8 (1984) 234-257.

[54] C. Rodrigo, F. Gaspar, X. Hu and L. Zikatanov, Stability and monotonicity for some discretizations of the Biot's consolidation model. Comput. Methods Appl. Mech. Eng. 298 (2016) 183-204.

[55] O.J. Sutton, The virtual element method in 50 lines of MATLAB. Numer. Algorithms 75 (2016) 1141-1159.

[56] X. Tang, Z. Liu, B. Zhang and M. Feng, A low-order locking-free virtual element for linear elasticity problems. Comput. Math. Appl. 80 (2020) 1260-1274.

[57] K. Terzaghi, Theoretical Soil Mechanics. John Wiley \& Sons, New York (1943).

[58] G. Vacca, Virtual element methods for hyperbolic problems on polygonal meshes. Comput. Math. Appl. 74 (2017) 882-898.

[59] G. Vacca and L. Beirão da Veiga, Virtual element methods for parabolic problems on polygonal meshes. Numer. Methods Part. Differ. Equ. 31 (2015) 2110-2134.

[60] P. Vermeer and A. Verruijt, An accuracy condition for consolidation by finite elements. Int. J. Numer. Anal. Methods Geomech. 5 (1981) 1-14.

[61] S.-Y. Yi, A coupling of nonconforming and mixed finite element methods for Biot's consolidation model. Numer. Methods Part. Differ. Equ. 29 (2013) 1749-1777.

[62] S.-Y. Yi, A study of two modes of locking in poroelasticity. SIAM J. Numer. Anal. 55 (2017) 1915-1936.

[63] B. Zhang and M. Feng, Virtual element method for two-dimensional linear elasticity problem in mixed weakly symmetric formulation. Appl. Math. Comput. 328 (2018) 1-25.

[64] B. Zhang, Y. Yang and M. Feng, Mixed virtual element methods for elastodynamics with weak symmetry. J. Comput. Appl. Math. 353 (2018) 49-71.

[65] O.C. Zienkiewicz and T. Shiomi, Dynamic behaviour of saturated porous media; the generalized Biot formulation and its numerical solution. Int. J. Numer. Anal. Methods Geomech. 8 (1984) 71-96. 